\title{
DATOS SOBRE LA EVOLUCIÓN DE LOS 'FRESNALES' O FRESNEDAS DE FLOR VALENCIANAS, OBTENIDOS MEDIANTE EL INVENTARIADO DIACRÓNICO
}

\author{
E. Laguna Lumbreras \\ Generalitat Valenciana, Centro para la Investigación y Experimentación Forestal \\ Avda. Comarques del País Valencià, 114. 46930 Valencia \\ laguna_emi@gva.es \\ P.P. Ferrer Gallego \\ Generalitat Valenciana, VAERSA \\ Avda. Corts Valencianes, $n^{\circ}$ 20. 46015 Valencia \\ flora.cief@gva.es \\ D.C. Gómez Montblanch \\ Universidad de Sevilla \\ C/ Doña María de Padilla, s/n. 41004 Sevilla \\ davidcristel@gmail.com

\section{Universidad del País Vasco \\ C/ Tomás y Valiente s/n. 01006 Vitoria-Gasteiz \\ pedrojose.lozano@ehu.es \\ guillermo.meatza@gmail.com} \\ P.J. Lozano Valencia y G. Meaza Rodríguez
}

\begin{abstract}
Resumen: Se exponen resultados del seguimiento de inventarios fitosociológicos de bosques de fresno de flor (Fraxinus ornus L.) valencianos, Hedero belicis-Cytisetum patentis fraxinetosum orni, monitorizados durante 3 décadas, cuyos datos de abundancia-dominancia se tomaron cada 10 años -1984,1994, 2004 y 2014- en la localidad del typus nominis de esta asociación vegetal en los montes de Malacara (Buñol, Valencia, España). $\mathrm{El}$ análisis de los datos demuestra un incremento progresivo de la riqueza específica y la cobertura media, notablemente para las especies conside-
\end{abstract}

Recibido: 05-12-2014. Aceptado: 12-06-2015. 
radas indicadoras de la evolución al encinar climácico local (Hedero belicis-Quercetum rotundifoliae).

Palabras clave: inventario de vegetación, método diacrónico, fresnedas de flor, España.

\begin{abstract}
Results on the monitoring of phytosociological relevees of Valencian manna ash (Fraxinus ornus L.) forest -Hedero belicis-Cytisetum patentis fraxinetosum orni- for 3 decades are shown; their abundancedominance data where collected every 10 years -1984, 1994, 2004 and 2014- in the typus nominis site of this plant association in Malacara Mountains (Buñol, Valencia, Spain). The data analysis demonstrate the progressive global increase in species richness and mean cover, notably for the species taken as evolution indicators towards the local, climacic helm oak forest (Hedero belicis-Quercetum rotundifoliae).
\end{abstract}

Keywords: vegetation relevee, diachronical method, manna ash forests, Spain.

\title{
1. Introducción
}

El desarrollo de estudios diacrónicos de la vegetación, particularmente cuando resulta conveniente el análisis de inventarios seriados tomados sobre parcelas fijas, acusa una notable escasez de trabajos disponibles con un período suficientemente amplio de seguimiento, a pesar de su relevancia para los estudios biogeográficos (v. Braun-Blanquet, 1979; Pintó et al., 2000). Salvo algunas excepciones notables -p.ej., Cardona, 1980- apenas existen antecedentes de trabajos en los que se haya realizado el inventariado vegetal sistemático de parcelas fijas por períodos suficientemente largos en España. Como parte del desarrollo de la tesis doctoral de Laguna (1995) se inventariaron las comunidades vegetales que forman parte de la serie de vegetación del encinar valenciano de media montaña-ver caracterización más adelante-, estableciéndose parcelas de seguimiento que han vuelto a revisitarse para su inventariado cada 10 años. En el presente artículo se analizan los resultados obtenidos para el caso de los 'fresnales', nombre local de la fresnedas de flor -formaciones forestales de ladera o fondo de barranco dominadas por la especie Fraxinus ornus L.- en la parte central de la provincia de Valencia.

\section{2. Área de estudio}

La zona objeto del estudio la constituye el paraje de la Umbría del Fresnal (Buñol, Valencia), que conforma la hemicuenca septentrional del Barranco del Fresnal, en el 
extremo occidental de la Sierra de Malacara o Montes de Buñol, en la provincia de Valencia. Esta zona ha sido descrita por Laguna (1997) por lo que aquí se aportan exclusivamente datos sintéticos, sin indicación detallada de las obras consultadas para cada componente ambiental, salvo las derivadas de trabajos de publicación más reciente.

La Umbría del Fresnal se encuentra íntegramente incluida en la celda UTM de $10 \mathrm{~km}$ de lado $30 \mathrm{~S}$ XJ77 -ver fig. $\mathrm{n}^{\mathrm{o}} 1-\mathrm{y}$ posee una superficie de 204 ha, con cotas comprendidas entre 605 (Hondo del Fresnal, XJ7565) y 964 m.s.n.m. (Pico del Retamar, XJ7764). El Barranco del Fresnal nace en el paraje del Collado Umán (XJ7865, 812 m.s.n.m.) y discurre en sentido SE-NW -fig. $n^{\circ} 2-$, por lo que la hemicuenca de aguas de umbría

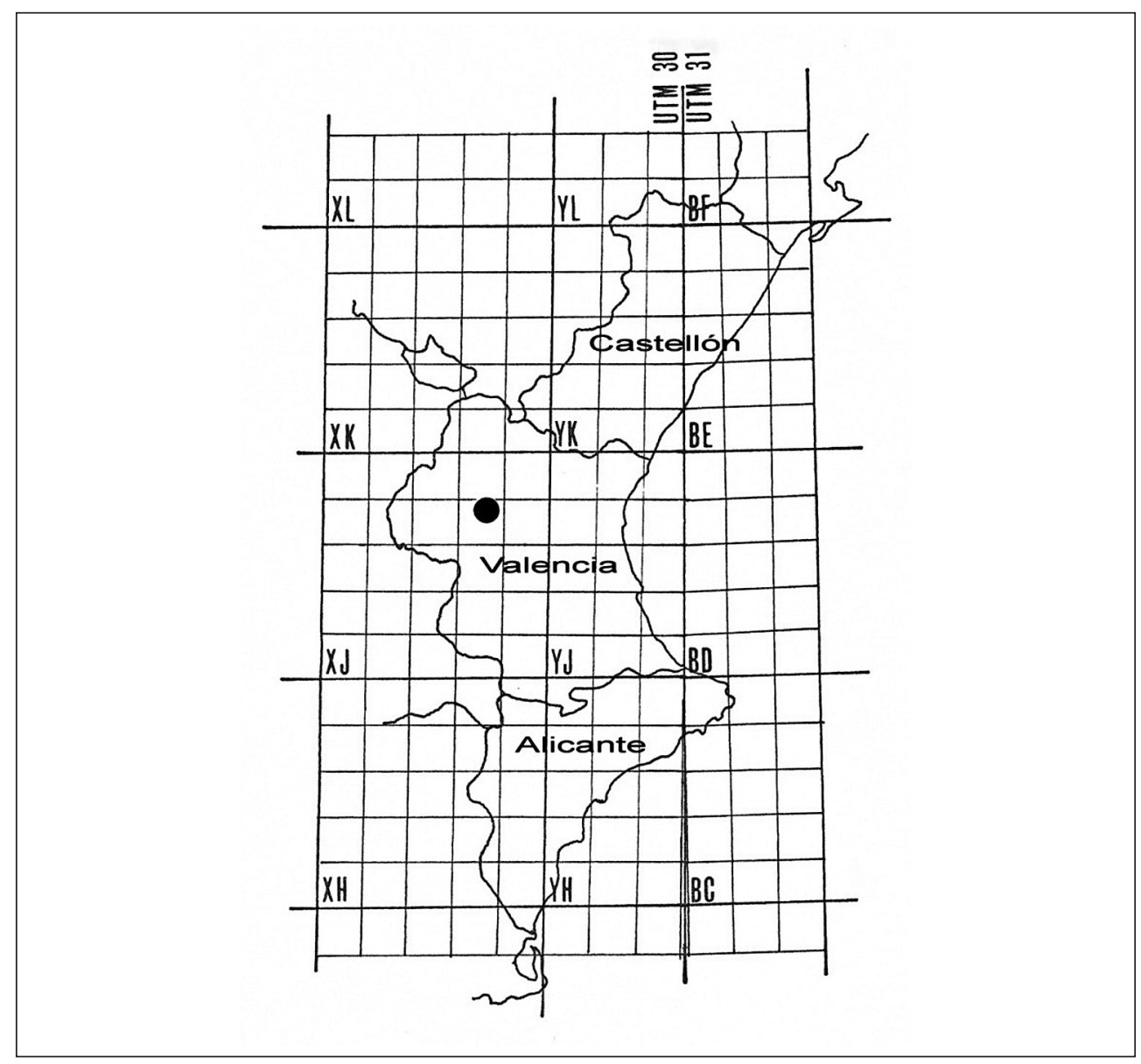

Figura 1. Ubicación del área de estudio dentro de la Comunidad Valenciana con sobreimpresión del retículo UTM en celdas de $20 \times 20 \mathrm{~km}$. 


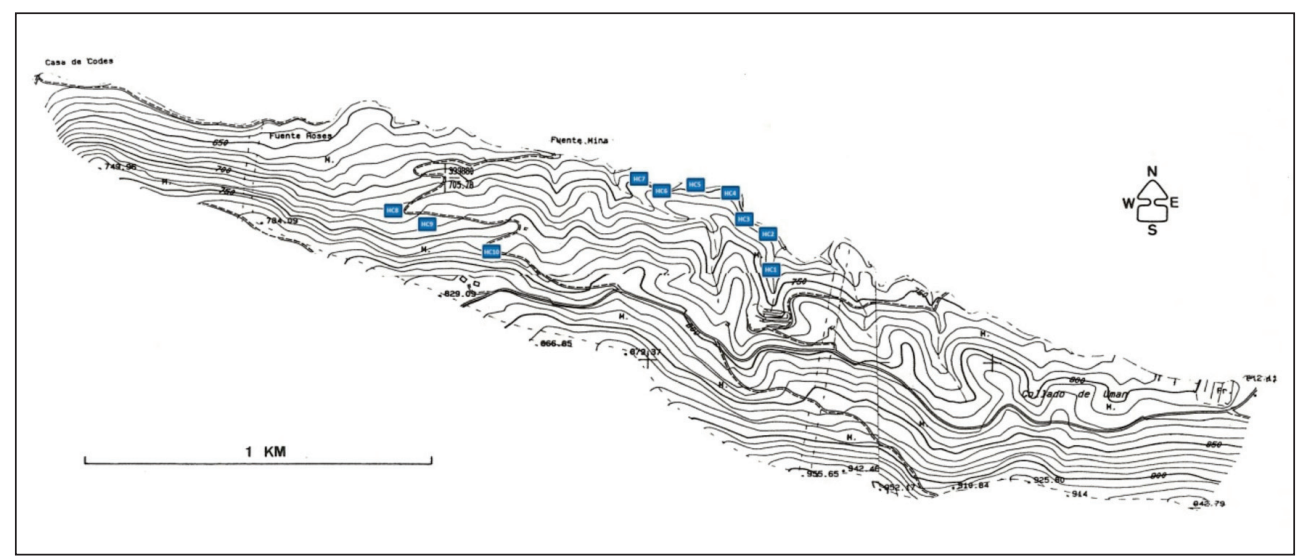

Figura 2. Topografía de la zona de estudio (isolíneas cada $10 \mathrm{~m}$ ). En cuadrados resaltados, ubicación de las parcelas utilizadas para el seguimiento diacrónico de la vegetación.

se encuentra orientada a NNE y la contraria -Solana del Arca-, a SSW. Los terrenos son de naturaleza básica, siendo dominantes los afloramientos del Cretácico -calcarenitas del Cenomaniense, dolomías sacaroideas del Cenomaniense-Turoniense y calizas gris-claro del Santoniense- y Neógeno -areniscas de matriz calcárea. Los suelos predominantes de la clasificación FAO-2006 (IUSS Grupo de Trabajo WRB, 2007) son los Luvisoles crómicos y cálcicos, Regosoles, Cambisoles cálcicos y crómicos, Rendzinas y Kastanozems. Desde el punto de vista bioclimático, considerando la clasificación adoptada por RivasMartínez et al. (2001), la zona corresponde al termoclima Mesomediterráneo superior y ombroclima seco-subhúmedo, con tendencia al Supramediterráneo subhúmedo por efecto de la intrazonalidad que aporta la orientación de la ladera. Siguiendo la clasificación corológica de los mismos autores (Rivas-Martínez et al., 2001) la zona de estudio se sitúa en el extremo nordoccidental del sector Setabense, dentro de la subprovincia Valenciana de la provincia Catalano-Provenzal-Balear. La fig. $\mathrm{n}^{-} 3$ expone la situación de la zona de estudio respecto de la sectorización propuesta por Costa $(1986,1999)$, recientemente modificada por Rivas-Martínez (2007) al transferir a la provincia CatalanoProvenzal-Balear la parte NE del antiguo distrito Setabense -D1 en la fig. n⿳0 3-, que pasa a denominarse subsector Turiano-Huertano, pero que no abarca la zona de estudio aquí analizada. Dada su situación dentro del sector Setabense, la Umbría del Fresnal posee una fuerte influencia de otros tres sectores colindantes: Valenciano-Tarraconense, Manchego y Maestracense -fig. $n^{-}$3-. El trabajo de Laguna (1997) facilita la relación de indicadores de estos sectores florísticos.

Dentro de la Umbría del Fresnal se han localizado poblaciones de 583 táxones de flora vascular hasta grado de subespecie, siendo predominante el elemento corológico Mediterráneo (73,6\% de los táxones). Aunque la forma etológica más representada son los te- 


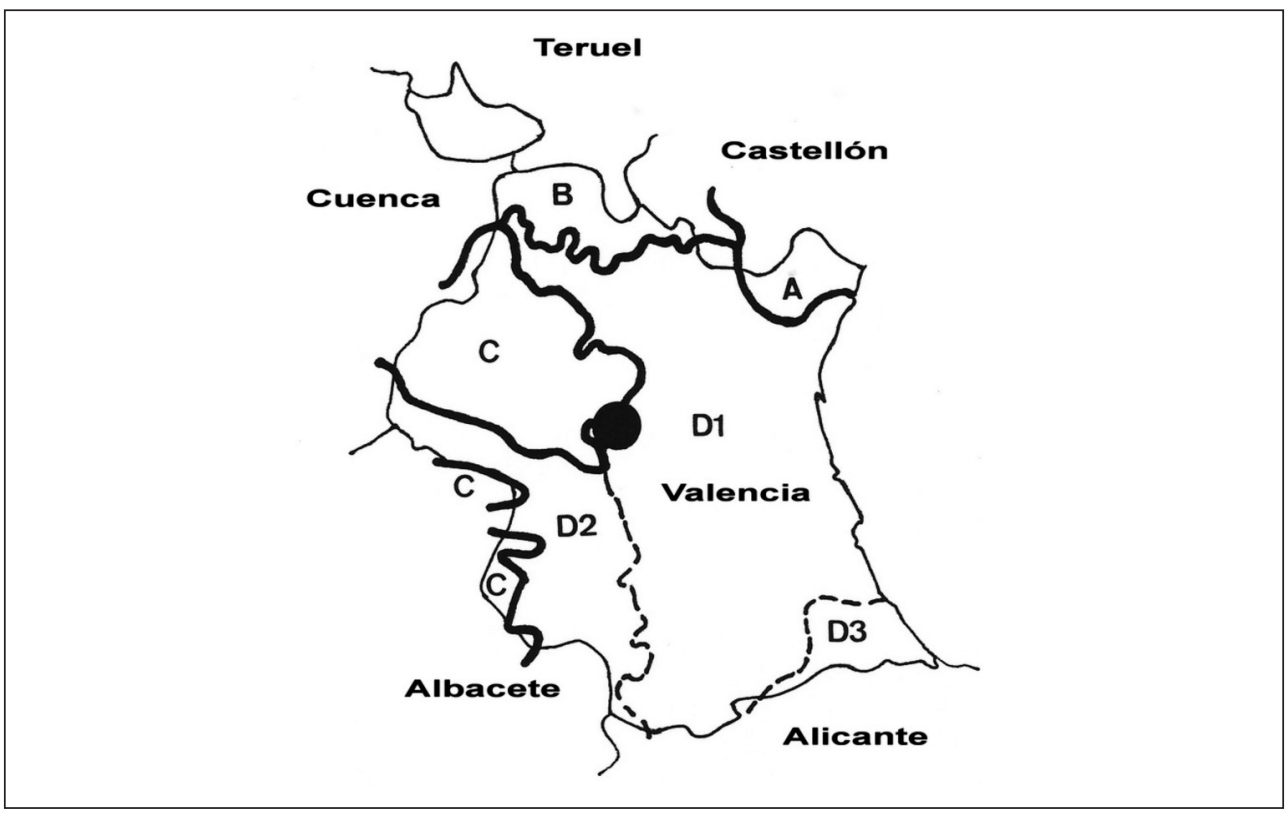

Figura 3. Sectorización corológica de la provincia administrativa de Valencia y sus contactos con otras limítrofes. Elaboración propia, a partir de los datos de Costa (1986, 1999). Para la escala territorial, ver la fig. $n^{\circ}$ 1. A: Sector Valenciano-Tarraconense; B: Maestracense; C: Manchego;

D: Setabense (D1: Subsector y Distrito Setabense; D2: Subsector Cofrentino-Villenense, Distrito Ayorano-Cofrentino; D3: Subsector Alcoyano-Dianense, Distrito Dianense.

rófitos o plantas anuales de porte herbáceo (33,1\% de los táxones), el paisaje local está extensivamente dominado por árboles y grandes arbustos. El territorio se encontraba fuertemente desertizado a principios del siglo XX como consecuencia de la sobreexplotación de los recursos vegetales, repoblándose en etapas sucesivas entre 1900 y 1917 con empleo de especies locales - Pinus halepensis, P. pinaster, P. nigra subsp. salzmanni- a partir de semilla de los escasos rodales remanentes de estas especies en la Sierra de Malacara; la incorporación de la zona de estudio al Catálogo de Montes de Utilidad Pública a finales del siglo XIX, la repoblación forestal, y las limitaciones al pastoreo y la extracción de madera por motivos de prevención hidrológico-forestal, favorecieron el rebrote de las frondosas preexistentes y su progresiva expansión, dando lugar mayoritariamente a un bosque multiestratificado dominado en su dosel superior por Pinus halepensis en torno a 20-25 m de altura, y en el intermedio -hasta los 8-10 m, aunque excepcionalmente se alcanzan los $20 \mathrm{~m}$ con ejemplares monumentales- por Quercus ilex subsp. rotundifolia, Q. faginea subsp. faginea y Fraxinus ornus, que de modo puntual y disperso conforman rodales de encinar. Los pinares locales se han adscrito a la comunidad Rhamno lycioidis-Quercetum cocciferae Br.-Bl. y O. Bolòs 1954, y los encinares al Hedero helicis- 
Quercetum rotundifoliae Costa, Peris y Stübing 1987, aunque en los enclaves más evolucionados tienden a dominar las especies caducifolias y marcescentes (Quercus faginea, Fraxinus ornus, Acer opalus subsp. granatense) propias del quejigar setabense Fraxino orni-Quercetum fagineae Rivas Goday y Borja in Rivas Goday et al. 1960 corr. Rivas-Martínez 1972. Los tipos de vegetación dominantes a nivel paisajístico en la zona de estudio se exponen en la fig. $\mathrm{n}^{-}$4; los protólogos de los binómenes fitosociológicos allí indicados corresponden a la revisión de Rivas-Martínez et al. (2001).

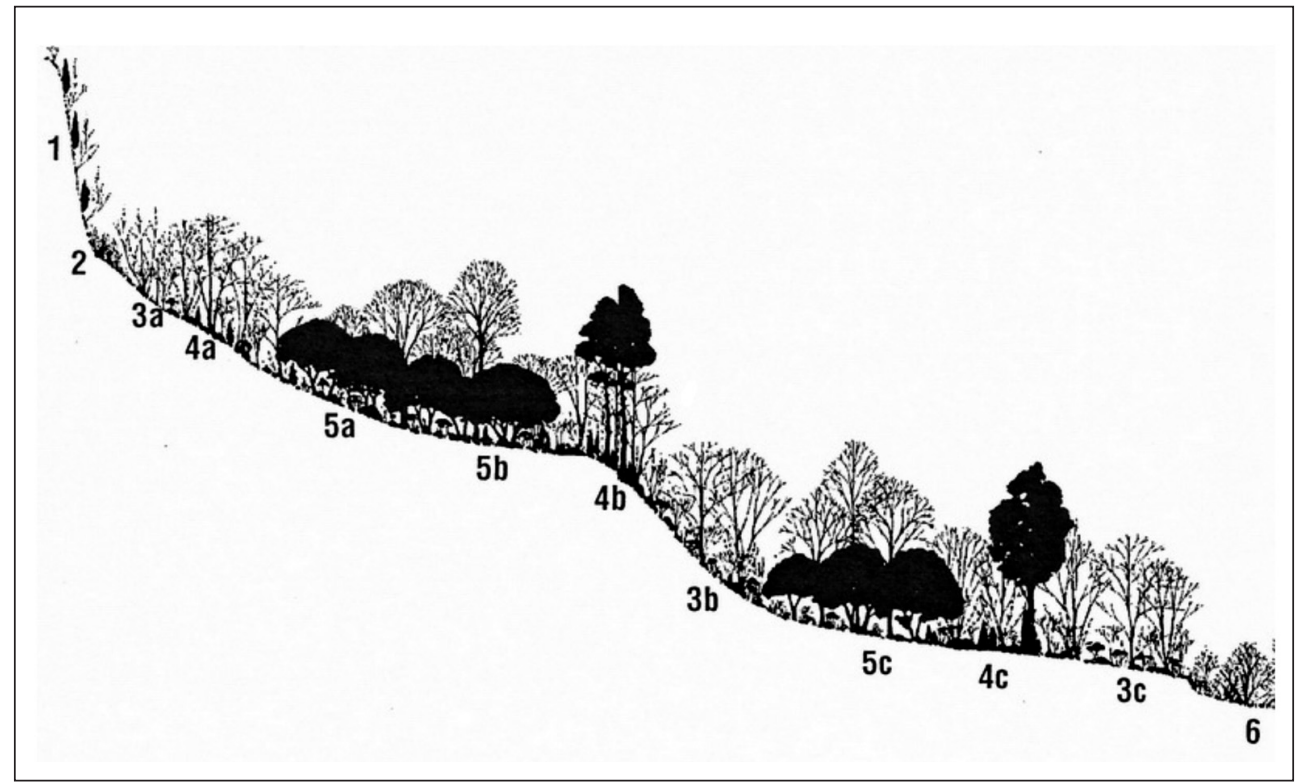

Figura 4. Representación sinóptica de la cliserie de comunidades vegetales presentes en la Umbría del Fresnal, con facies o variantes de cinto rocoso (a), media ladera (b) y fondo de barranco (c). 1: Sabinares de roca (Rhamno-Juniperetum phoeniceae). 2: Zarzales dominados por Rubus ulmifolius y Osyris alba. 3: Fresnales (Hedero-Cytisetum patentis fraxinetosum orni).

4: Coscojares (Rhamno-Quercetum cocciferae) con Fraxinus ornus y/o Pinus halepensis.

5: Encinares y encinar-quejigares (Hedero helicis-Quercetum rotundifoliae) con F. ornus. 6: Espinal-zarzales de fondo de barranco (Rubo ulmifolii-Crataegetum brevispinae). Extraído de Laguna (1997: 86), reproducido con permiso.

La comunidad arbórea más relevante de la zona la constituyen los 'fresnales', nombre que reciben localmente las fresnedas de flor-dominadas por Fraxinus ornus-, por contraposición a las 'fresnedas' en sentido estricto, fitónimo que se reserva para las formaciones riparias dominadas por F. angustifolia (Laguna, 1997). Los fresnales o fresnedas de flor se consideran tipos de vegetación prácticamente endémicos de la Comunidad Valenciana dentro de la Península Ibérica. Teniendo en cuenta los bioclimas y sectorización corológica 
de Rivas-Martínez et al. (2001) estas comunidades están restringidas totalmente al sector Setabense, habiéndose definido hasta tres formaciones vegetales respectivamente correspondientes a los pisos bioclimáticos termo-, meso- y supramediterráneo: Viburno tiniFraxinetum orni Costa, Pérez-Badía y P. Soriano 1995, Hedero-Cytisetum patentis Mateo 1983 subass. fraxinetosum orni Mateo 1983, y Fraxino orni-Aceretum granatensis Alcaraz, Ríos, Solanas y M.B. Crespo 2001. La Umbría del Fresnal alberga el typus nominis o inventario de referencia del Hedero-Cytisetum patentis fraxinetosum orni, que constituye localmente la orla caducifolia propia de los fondos de barranco, las grandes pedrizas y los linderos en los márgenes de las pistas forestales más antiguas. La inversión térmica local y la consecuente tendencia a la dominancia puntual de especies de óptimo supramediterráneo, hace que la fresneda de flor se enriquezca en esta zona de especies del Fraxino orni-Aceretum granatensis (Laguna y Pérez Rocher, 1998).

Resumiendo lo indicado por Laguna (1997), la fresneda de flor es una comunidad multiestratificada de hasta 8-10 m de talla, con dosel arbóreo caducifolio dominado por Fraxinus ornus, a menudo compartido con Acer granatense y elementos de las etapas más maduras como Quercus ilex subsp. rotundifolia y Q. faginea; el estrato subarbóreo o arborescente está dominado por una mezcla de caducifolios de hasta 3-4 m -principalmente Cytisus patens y Amelanchier ovalis- y perennifolios como Viburnum tinus y Arbutus unedo. El estrato arbustivo y el herbáceo poseen una mezcla de especies heliófilas y esciófilas donde usualmente dominan indicadores de enclaves umbrosos y suelos más o menos profundos (Ononis aragonensis, Brachypodium phoenicoides, Festuca fenas subsp. arundinacea, etc.). La diferencia con el coscojar (Rhamno-Quercetum rotundifoliae), localmente arbolado a menudo con P. halepensis y formando una facies local donde está enriquecido con frondosas como el propio F. ornus, A. granatense, etc., estriba en la mayor dominancia de Quercus coccifera en aquella comunidad -fig. $\mathrm{n}^{\mathrm{O}}$ 5-.

La serie de vegetación para la zona de estudio corresponde al Hedero belicis-Querceto rotundifoliae sigmetum, formulado junto a la propia comunidad potencial ya indicada, en el artículo descriptivo original (Costa et al., 1987), pero inicialmente restringido al sector Valenciano-Tarraconense de vegetación. Su extensión al sector Setabense fue asentada por Ballester y Stübing (1990). Aunque parece evidente la existencia de una comunidad característica de este sector, que se ha indicado como subasociación fraxinetosum orni (Laguna, 1995), no ha sido formalmente descrita. La principal diferencia entre los encinares -localmente denominados 'carrascares'- continentales (Quercetum rotundifoliae Br.-Bl. y O. Bolòs in Vives 1956) y el Hedero helicis-Quercetum rotundifoliae radica en que en este último la etapa de degradación inmediata no es necesariamente un coscojar de Quercus coccifera, sino formaciones mixtas o vegetaciones de orla caducifolia, que en el sector Setabense corresponderían al Hedero belicis-Cytisetum patentis fraxinetosum orni (Ballester y Stübing, 1990). Paralelamente, este tipo de encinar posee una elevada cobertura de hiedra (Hedera helix), con valores fitosociológicos de abundancia-dominancia usualmente mayores de 3, tanto en la cobertura de tallos vegetativos del estrato herbáceo, como de los vástagos florales en el arbóreo. Para el sector 

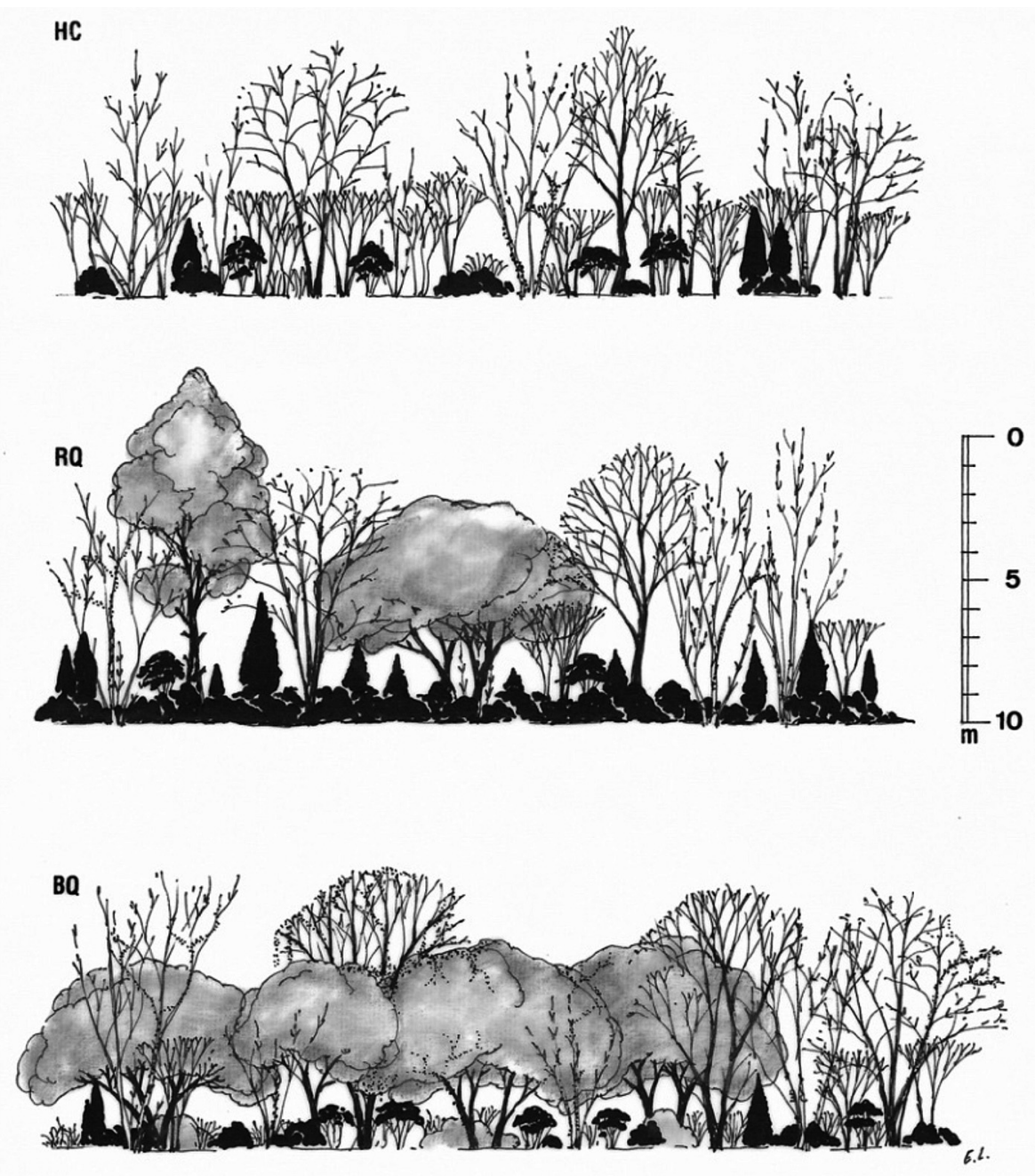

Figura 5. Esquema de la estructura de la vegetación en formas óptimas de las comunidades vegetales del área de estudio dominadas por frondosas. HC: Hedero-Cytisetum patentis fraxinetosum orni. RQ: Rhamno-Quercetum rotundifoliae (facies local con Fraxinus ornus). BQ: Hedero helicis-Quercetum rotundifoliae (facies local con F. ornus). Extraído de Laguna (1997: 110), reproducido con permiso.

Setabense, la hiedra estaría representada fundamentalmente por la subsp. rbizomatifera, considerada como endemismo ibérico. Esta misma subespecie tiende a presentar igualmente alta cobertura en los fresnales de flor, pero más restringida al estrato herbáceo. 
Conforme a nuestras observaciones en la zona de estudio, en el caso de las etapas sucesionales progresivas, la hipótesis más razonable sería que el encinar con fresno de flor pudiera provenir directamente de la evolución de los fresnales y/o de los coscojares con fresno de flor, e incluso que la expansión de Q. coccifera pudiera facilitar la conversión de fresnales en coscojares. A largo plazo esta evolución sucesional se podría dar en la vegetación de ladera, pero también en fondos de barranco si no ocurrieran a largo plazo fuertes episodios de lluvias torrenciales que ralentizaran el proceso -fig. nํ 6-.

\section{Metodología}

A raíz del inventariado de diversas unidades de vegetación abordado en 1984 para el desarrollo de la tesis doctoral de Laguna (1995), y una vez acabada la fase de seguimiento en campo allí abordada, se decidió inventariar regularmente las parcelas de algunas de las comunidades mediante visitas a realizar un año de cada 10, abordándose en consecuencia en 1994, 2004 y 2014.

Para el presente estudio se han muestreado 10 parcelas de la comunidad Hedero-Cytisetum patentis fraxinetosum orni -fig. $\mathrm{n}^{-}$2- cuyos datos básicos se exponen en la tabla $\mathrm{n}^{\mathrm{o}}$ 1, y que corresponden a las parcelas de seguimiento del trabajo ya citado (Laguna, 1995). Cada parcela se inventarió en los años 1984, 1994, 2004 y 2014, realizándose al menos 3 visitas a cada parcela - primaveral, estival y otoñal- a fin de cubrir suficientemente la identificación de todas las especies, incluidas las de presencia temporal más esporádica como los terófitos efímeros. En cada visita se tomaron datos de la abundancia-dominancia de todas las especies presentes siguiendo el método de Braun-Blanquet (1979). Para los tratamientos numéricos más abajo detallados, los 6 rangos de estimación de abundancia-dominancia $(+, 1,2,3,4$ y 5$)$ se expresaron a través de sus valores medios de cobertura de Tüxen-Ellenberg (v. Braun-Blanquet, 1979: 38 y 50), que corresponden respectivamente a los valores $0,001,0,05,0,175,0,375,0,625$ y 0,875 , expresables igualmente mediante los porcentajes de cobertura $0,1 \%, 5 \%, 17,5 \%, 37,5 \%, 62,5 \%$ y $87,5 \%$.

En los muestreos se realizaron 2 tipos de inventarios fitosociológicos para cada parcela. De un lado se acometió el inventariado tradicional, donde cada especie está representada por un único valor - de + a 5, según lo ya indicado-. Por otro, se realizó un inventario por estratos, donde cada especie tiene un valor para la cobertura que alcanza en cada uno de los siguientes 4 estratos: arbóreo, de 3 a $8 \mathrm{~m}$ sobre el nivel del suelo; subarbóreo, de 1 a $3 \mathrm{~m}$; arbustivo de 0,3 a $1 \mathrm{~m}$; y herbáceo, de 0 a 0,3 m. Por ejemplo, en una misma parcela $F$. ornus poseerá 4 valores de cobertura estratificada, correspondientes respectivamente a los adultos de gran talla con copas en el estrato arbóreo, subadultos o adultos jóvenes del estrato subarbóreo, juveniles del arbustivo, y plántulas y pequeños juveniles del herbáceo. 


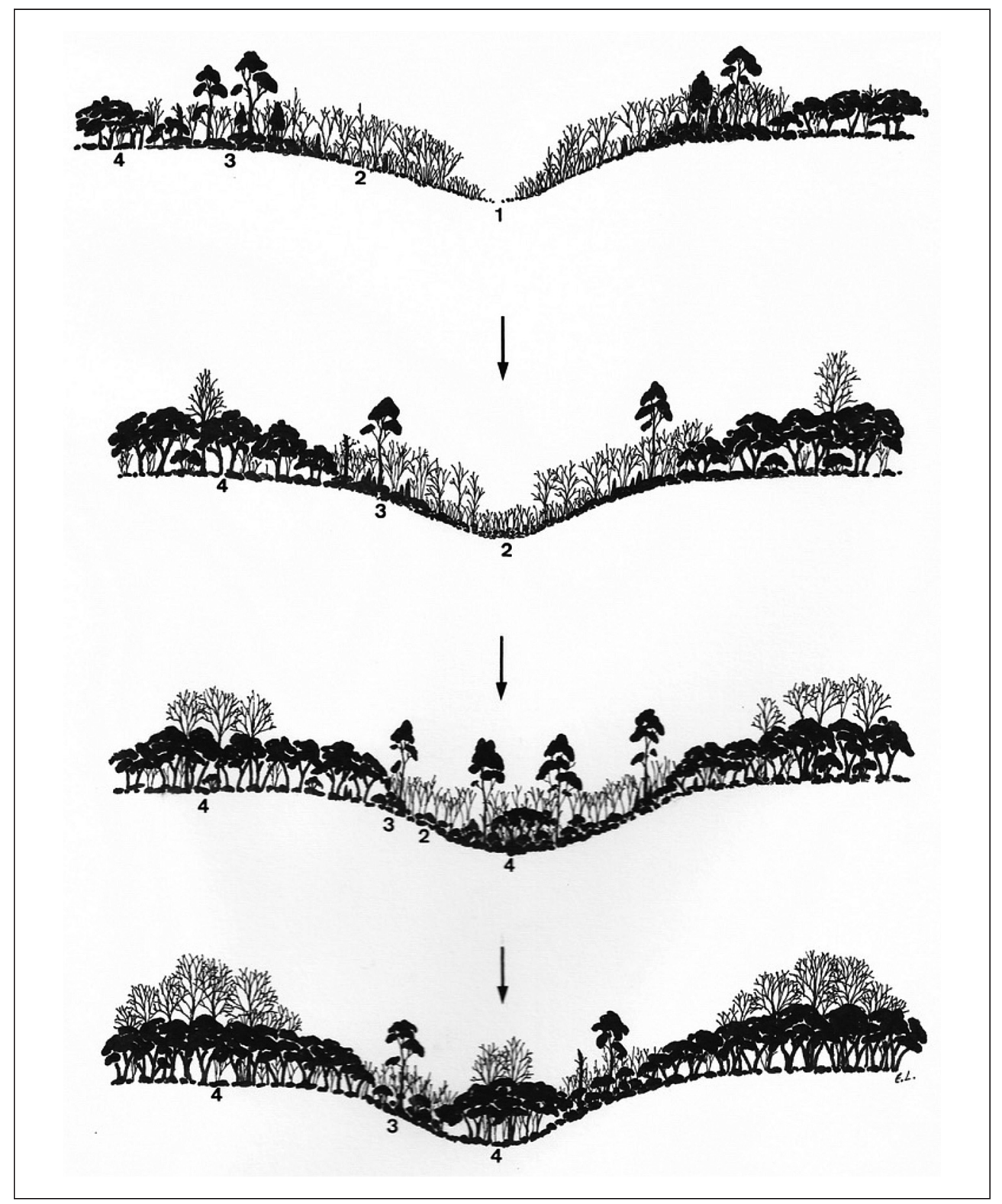

Figura 6. Esquema del avance de la sucesión vegetal local en laderas y barrancos de umbría que vierten al Barranco del Fresnal. 1: Vegetaciones herbáceas. 2: Hedero-Cytisetum patentis fraxinetosum orni. 3: Rhamno-Quercetum rotundifoliae (facies local con Fraxinus ornus). 4: Hedero helicis-Quercetum rotundifoliae (facies local con F. ornus y Quercus faginea). Extraído de Laguna (1990: 69), reproducido con permiso. 
Tabla 1. Datos de caracterización de las parcelas inventariadas

\begin{tabular}{|l|c|c|c|c|c|c|c|c|c|c|}
\hline Nombre parcela & HC1 & HC2 & HC3 & HC4 & HC5 & HC6 & HC7 & HC8 & HC9 & HC10 \\
\hline Tipo & $\mathrm{A}$ & $\mathrm{A}$ & $\mathrm{A}$ & $\mathrm{A}$ & $\mathrm{A}$ & $\mathrm{A}$ & $\mathrm{A}$ & $\mathrm{B}$ & $\mathrm{B}$ & $\mathrm{B}$ \\
Altitud & 726 & 707 & 705 & 701 & 699 & 688 & 685 & 758 & 762 & 755 \\
Orientación & $\mathrm{NNW}$ & $\mathrm{N}$ & $\mathrm{N}$ & $\mathrm{NNE}$ & $\mathrm{N}$ & $\mathrm{NNE}$ & $\mathrm{NE}$ & $\mathrm{NNW}$ & $\mathrm{N}$ & $\mathrm{N}$ \\
Inclinación & 5 & $<5$ & $<5$ & $<5$ & $<5$ & $<5$ & $<5$ & $<5$ & $<5$ & $<5$ \\
Superficie & 90 & 104 & 100 & 105 & 88 & 90 & 100 & 96 & 90 & 90 \\
Altura & 4,5 & 6,5 & 7,0 & 7,5 & 6,0 & 4,4 & 4,5 & 6,0 & 7,0 & 5,5 \\
\hline
\end{tabular}

Leyenda: Tipo: A: Fondo de barranco; B: Orla forestal de ladera; Altitud: Altitud sobre el nivel del mar, en m; Inclinación: Inclinación media en grados sexagesimales; Superficie: Superficie en $\mathrm{m}^{2}$; Altura: Altura máxima de la vegetación al inicio del seguimiento (año 1984), en $m$.

A fin de realizar una aproximación a la caracterización florística y estructural de las fresnedas de flor, tanto para cada parcela como para cada estrato dentro de la parcela se calcularon dos valores:

- Riqueza florística, correspondiente al número de especies.

- Cobertura solapada, en el sentido ya expuesto en los trabajos de Laguna (2012), Gómez-Montblanch et al. (2014a) y Laguna y Ferrer-Gallego (2015). Dicho concepto corresponde a la suma de los valores de cobertura media de Tüxen-Ellenberg para todas las especies presentes en un inventario, o en su caso en un estrato. Se denomina 'solapada', ya que al albergar el solape de copas, ramas u otras partes de las plantas de varias especies, en las parcelas o estratos densos puede superar con facilidad el valor 1, que correspondería al 100\% de cobertura.

A efectos de incrementar el conocimiento sobre la dinámica de la vegetación se han distinguido 3 tipos de especies en función de su relación con la sucesión vegetal local, descrita por Laguna (1995, 1997):

- R: Regresivas, propias de etapas previas (matorrales, maquias) o ambientes más secos, abiertos o iluminados.

- N: Neutras, que no pueden definirse fácilmente como regresivas o progresivas.

- P: Progresivas, propias de etapas sucesionales consideradas avanzadas (Hedero belicis-Quercetum rotundifoliae), ambientes nemorales, suelos con horizonte superior isohúmico -tendencia al Kastanozem- o de mayor humedad edáfica y ambiental.

Las plantas se han asignado a cada uno de estos 3 grupos sucesionales en función del comportamiento observado repetidamente en campo en la zona de estudio, detallado especialmente por Laguna (1995). Las especies localizadas y su adscripción a los 
3 grupos ( $\mathrm{R}, \mathrm{N}$ o P) figuran en el anexo $\mathrm{n}^{-}$1. Debe señalarse que la asignación a un grupo concreto no es extrapolable a zonas alejadas del área de estudio, ya que como suelen indicar los tratados básicos en biogeografía y fitosociología (v. Braun-Blanquet, 1979; Ozenda, 1964) los mismos táxones pueden poseer diferencias comportamentales en función de su ubicación en sus áreas de distribución, altitudes, topografía, materiales geológicos, etc.

Las comparaciones de datos se han realizado mediante el test del estadístico t de Student para el contraste de las medias en distribuciones de datos apareados (v. Sokal y Rohlf, 1994), usando el paquete estadístico PAST v. 1.56b (Hammer y Harper, 2006; Hammer et al., 2001).

Las especies se indican sin expresión del autor del binomen científico, al corresponder éste a los indicados en las obras Flora Valentina (Mateo et al., 2011-2015), Flora iberica (Castroviejo, 1986-2014), y el trabajo de Mateo y Crespo (2014), tomados por ese orden de prioridad en el caso de sinonimias.

\section{Resultados}

\subsection{Datos generales y resultados sin diferenciación de grupos sucesionales}

En los 10 inventarios se contabilizó la presencia de 137 especies, no estando representada ninguna de ellas por más de un taxon de nivel inferior excepto Lonicera implexa L., para la que Laguna (1995) había reconocido previamente por su diferente aspecto morfológico, fenología y comportamiento local la f. velutina Pau y la var. valentina (Pau ex Willk.) Maire [basiónimo: L. valentina Pau]; la primera incluye a plantas de hojas menos coriáceas que las típicas subsp. implexa, con envés villoso-velutino en los pares de hojas medios y superiores; la segunda corresponde a plantas de baja talla, usualmente no villosas, moderadamente coriáceas y con hojas pequeñas y numulariformes. Todos lo táxones correspondían a especies puras excepto Quercus $\times$ auzandrii nothosubsp. agrifolia (Q. coccifera $\times$ Q. ilex subsp. rotundifolia) y Q. $\times$ battandieri $(Q$. coccifera $\times Q . f a$ ginea). De los 137 táxones, se consideraron regresivos 34 (24,82\% del total), neutros 41 $(29,92 \%)$ y 62 progresivos $(45,26 \%)$-ver anexo $\mathrm{n}^{\mathrm{o}} 1-$. Los datos de frecuencia y cobertura media obtenidos para cada una de las especies se exponen en el anexo $\mathrm{n}^{\mathrm{o}} 2$.

Los resultados generales de riqueza y cobertura solapada para cada año, con los promedios de las 10 parcelas, se expresan en la tabla $\mathrm{n}^{\circ} 2$ y se ilustran en la fig. $\mathrm{n}^{-}$7. Para el caso del número de especies, los 3 estratos superiores exhiben un progresivo incremento de sus valores medios -sin merma de que sus diferencias interanuales pueden ser poco o no significativas-, pero no ocurre lo mismo con el estrato herbáceo y con el inventario unificado para los 4 estratos, que presenta un valor superior en el 
Tabla 2. Datos medios y desviaciones típicas de la riqueza (número de táxones) y cobertura solapada obtenidos de los inventarios $(n=10)$ para el inventario no diferenciado

en estratos (multiestratificado) y para los correspondientes a cada uno de los 4 estratos en los 4 años de muestreo

\begin{tabular}{|l|c|c|c|c|c|c|}
\hline \multirow{2}{*}{} & \multicolumn{3}{|c|}{ Año } & \multicolumn{2}{c|}{ Variación 2014-1984 } \\
\cline { 2 - 7 } & 1984 & 1994 & 2004 & 2014 & Neta & $\%$ Incr. \\
\hline Riqueza (No especies) & & & & & \\
\hline Multiestratificado & $40,2+/-4,984$ & $49,3+/-7,703$ & $48,1+/-6,887$ & $45,1+/-4,280$ & $4,9+/-5,907$ & 12,19 \\
Arbóreo & $3,8+/-0,980$ & $4,1+/-1,044$ & $4,7+/-1,345$ & $4,9+/-1,136$ & $1,1+/-1,135$ & 26,95 \\
Subarbóreo & $11,2+/-0,980$ & $13,2+/-1,887$ & $13,9+/-2,071$ & $15,3+/-1,418$ & $4,1+/-1,446$ & 36,61 \\
Arbustivo & $13,3+/-2,002$ & $17,7+/-2,722$ & $17,8+/-1,470$ & $18,3+/-1,792$ & $5,0+/-2,683$ & 37,59 \\
Herbáceo & $33,7+/-5,041$ & $43,0+/-6,261$ & $42,9+/-4,825$ & $39,0+/-5,780$ & $5,5+/-5,780$ & 16,32 \\
\hline Cobertura solapada & & & & & & \\
\hline Multiestratificado & $2,060+/-0,497$ & $2,696+/-0,872$ & $3,506+/-0,797$ & $3,720+/-1,003$ & $1,660+/-0,826$ & 80,58 \\
Arbóreo & $0,714+/-0,311$ & $0,871+/-0,423$ & $1,184+/-0,412$ & $1,356+/-0,489$ & $0,642+/-0,241$ & 89,91 \\
Subarbóreo & $0,521+/-0,242$ & $0,920+/-0,308$ & $1,306+/-0,415$ & $1,419+/-0,440$ & $0,898+/-0,419$ & 172,36 \\
Arbustivo & $0,184+/-0,107$ & $0,463+/-0,169$ & $0,679+/-0,244$ & $0,835+/-0,348$ & $0,670+/-0,393$ & 364,13 \\
Herbáceo & $0,849+/-0,268$ & $1,268+/-0,501$ & $1,737+/-0,382$ & $1,723+/-0,468$ & $0,874+/-0,431$ & 102,94 \\
\hline
\end{tabular}

En el número de especies se ha reducido el número de decimales en el valor medio, al corresponder cada dato al promedio de 10 valores enteros. En el bloque de columnas a la derecha se expresa la diferencia de los valores obtenidos entre 2014 y 1984, indicada en valor neto de riqueza o cobertura, como en porcentaje de incremento obtenido (\%Incr.).

tramo intermedio, hacia 1994 y 2004. En todos los casos los valores medios al final del seguimiento, en 2014, son superiores a los de la etapa inicial en 1984. No ocurre así con la cobertura solapada, donde los datos ilustran una tendencia al incremento progresivo en todos los estratos y el inventario unificado -fig. $\mathrm{n}^{-}$7-, con la excepción poco significativa del estrato herbáceo para la transición de 2004 a 2014, donde el valor alcanzado puede considerarse prácticamente estabilizado.

Las diferencias comportamentales se aprecian además con los valores obtenidos por el estadístico t para la comparación de los datos interdecenales de un mismo estrato, o en su caso los del inventario multiestratificado. Como puede observarse en la tabla no $\mathrm{n}^{-}$, el distanciamiento es mayor en los datos de cobertura, en correspondencia con las gráficas, existiendo muy pocos casos donde las medias de muestreos sucesivos no sean significativamente distintas, y alcanzándose además unos valores de p marcadamente inferiores cuando se comparan los valores inicial y final de la serie temporal. Por el contrario, la misma tabla permite ver cómo los valores de la riqueza específica se incrementaron sustancialmente en el primer paso decenal -de 1984 a 1994-, y aunque en todos los ca- 


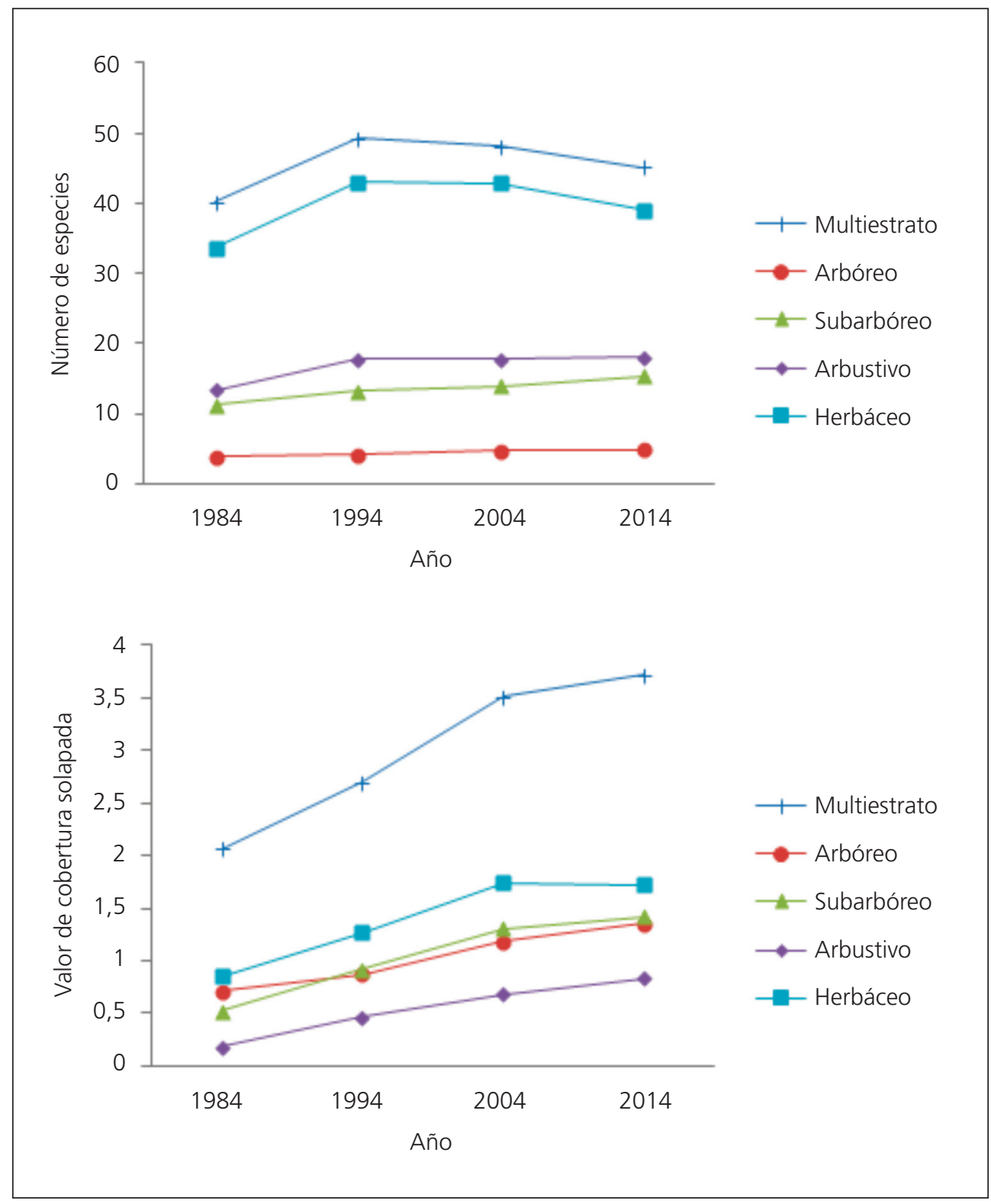

Figura 7. Representación de la evolución interdecenal del número de especies y del valor de cobertura solapada. 
Tabla 3. Valores del estadístico $t(n=10, g d l=9)$ para el contraste de la media de datos apareados entre los períodos de muestreo dentro de cada estrato y en el inventario multiestratificado

\begin{tabular}{|l|c|c|c|c|}
\hline $\begin{array}{l}\text { Valores absolutos de } t \\
\text { por estratos }\end{array}$ & 1984 vs 1994 & 1994 vs 2004 & 2004 vs 2014 & 1984 vs 2014 \\
\hline Riqueza (No especies) & & & & \\
\hline Multiestratificado & $4,622^{* * *}$ & 0,739 n.s. & 2,170 n.s. & $2,489^{*}$ \\
Arbóreo & 1,964 n.s. & 1,765 n.s. & 1,000 n.s. & $2,905^{*}$ \\
Subarbóreo & $4,243^{* *}$ & $2,333^{*}$ & $2,941^{*}$ & $8,508^{* * * *}$ \\
Arbustivo & $5,202^{* * *}$ & 0,111 n.s. & 0,889 n.s. & $5,590^{* * * *}$ \\
Herbáceo & $4,411^{* *}$ & 0,078 n.s. & $3,422^{* *}$ & $2,751^{*}$ \\
\hline Cobertura solapada & & & & \\
\hline Multiestratificado & $4,379^{* * *}$ & $7,061^{* * * *}$ & 1,292 n.s. & $6,346^{* * * *}$ \\
Arbóreo & $2,861^{*}$ & $5,516^{* * *}$ & $2,379^{*}$ & $8,419^{* * * * *}$ \\
Subarbóreo & $6,545^{* * *}$ & $7,557^{* * * *}$ & 1,259 n.s. & $6,776^{* * * * *}$ \\
Arbustivo & $4,724^{* * *}$ & $4,094^{* * *}$ & $3,084^{* *}$ & $5,394^{* * * *}$ \\
Herbáceo & $3,830^{* * *}$ & $4,978^{* * *}$ & 0,168 n.s. & $6,419^{* * * *}$ \\
\hline
\end{tabular}

Notación: Medias significativamente diferentes para los niveles $p=0,05(*), 0,01(* *), 0,001(* * *) \circ 0,0001(* * * *)$. n.s.: Medias significativamente no diferentes al $95 \%$ o más de confianza.

sos hay diferencia significativa entre el número de especies inicial y final, tanto para cada estrato como para los inventarios completos, la mayoría de datos para los pasos intermedios -de 1994 a 2004 y de 2004 a 2014- no exhiben tales diferencias. Ello permite considerar que se tendería a un valor asintótico en el número de especies, pero no puede asegurarse con la misma determinación para el caso de la cobertura solapada.

\subsection{Resultados con diferenciación de grupos sucesionales}

Los valores observados para la riqueza y la cobertura diferenciando los grupos de especies regresivas $(\mathrm{R})$, neutras $(\mathrm{N})$ y progresivas $(\mathrm{P})$ para la sucesión vegetal en la fresneda de flor, se muestran en la tab. no $\mathrm{n}^{\mathbf{0}}$. La única fila de la tabla con ausencia de datos es la de las especies del tipo $\mathrm{N}$ en el estrato arbóreo, ya que la reducida representación de especies de ese dosel estaba compuesta sólo por especies que se consideraron P o R. Los datos de la tabla $\mathrm{n}^{\circ} 4$ arrojan una información de particular interés, ya que mientras las especies del tipo $\mathrm{P}$ incrementan por cada decenio su número de especies y cobertura, tanto a nivel global como para cada estrato, en las de tipo R se observa claramente una dinámica inversa, demostrando el avance de la sucesión vegetal en los 
Tabla 4. Datos equivalentes a los expresados en la tabla $n^{\circ} 3$, pero referidos a los 3 tipos sucesionales de especies (regresivas: $\mathrm{R}$; neutras: $\mathrm{N}$; progresivas: $\mathrm{P}$ ) tanto dentro de cada estrato como en los inventarios multiestratificados

\begin{tabular}{|c|c|c|c|c|c|c|}
\hline & \multicolumn{4}{|c|}{ Año } & \multicolumn{2}{|c|}{ Variación 2014-1984 } \\
\hline & 1984 & 1994 & 2004 & 2014 & Neta & \%Incr. \\
\hline \multicolumn{7}{|c|}{ Riqueza (№ especies) } \\
\hline \multicolumn{7}{|l|}{ № especies $\mathrm{P}$} \\
\hline Multiestratificado & $17,0+/-3,232$ & $27,7+/-4,809$ & $34,0+/-5,164$ & $35,1+/-3,247$ & $18,1+/-5,646$ & 105,89 \\
\hline Arbóreo & $2,7+/-0,675$ & $3,0+/-0,816$ & $4,0+/-1,414$ & $4,5+/-1,179$ & $1,8+/-0,919$ & 66,67 \\
\hline Subarbóreo & $8,4+/-0,966$ & $10,5+/-1,509$ & $11,8+/-1,476$ & $13,4+/-0,966$ & $5,0+/-1,491$ & 59,52 \\
\hline Arbustivo & $8,0+/-1,491$ & $13,1+/-2,283$ & $15,8+/-1,619$ & $16,8+/-1,751$ & $8,8+/-2,440$ & 110,00 \\
\hline Herbáceo & $14,2+/-3,553$ & $25,9+/-4,557$ & $32,2+/-4,709$ & $31,4+/-2,989$ & $17,2+/-5,160$ & 121,13 \\
\hline \multicolumn{7}{|l|}{ № especies $\mathrm{N}$} \\
\hline Multiestratificado & $10,9+/-2,767$ & $12,6+/-2,914$ & $10,2+/-2,821$ & $7,8+/-2.044$ & $-3,1+/-2,424$ & $-28,44$ \\
\hline Subarbóreo & $1,8+/-0,789$ & $1,9+/-0,876$ & $1,6+/-0,843$ & $1,5+/-0,707$ & $-0,3+/-0,483$ & $-16,67$ \\
\hline Arbustivo & $1,5+/-1,179$ & $1,6+/-0,516$ & $1,1+/-0,994$ & $1,1+/-0,876$ & $-0,4+/-1,350$ & $-26,67$ \\
\hline Herbáceo & $10,0+/-2,494$ & $11,7+/-2,751$ & $8,8+/-2,700$ & $6,6+/-2,271$ & $-3,4+/-2,503$ & $-34,00$ \\
\hline \multicolumn{7}{|l|}{ № especies $\mathrm{R}$} \\
\hline Multiestratificado & $12,3+/-3,020$ & $8,9+/-1,663$ & $3,8+/-1,135$ & $2,2+/-1.033$ & $-10,1+/-3,107$ & $-82,11$ \\
\hline Arbóreo & $1,0+/-0,667$ & $1,1+/-0,738$ & $0,7+/-0,483$ & $0,4+/-0,516$ & $-0,6+/-0,516$ & $-60,00$ \\
\hline Subarbóreo & $1,0+/-0,667$ & $0,9+/-0,738$ & $0,5+/-0,707$ & $0,4+/-0.516$ & $-0,6+/-0,516$ & $-60,00$ \\
\hline Arbustivo & $3,8+/-1,317$ & $3,0+/-1,054$ & $0,8+/-0,632$ & $0,4+/-0,516$ & $-3,4+/-1,578$ & $-89,47$ \\
\hline Herbáceo & $9,2+/-1,989$ & $4,4+/-1,524$ & $1,9+/-0,994$ & $0,9+/-0,316$ & $-8,3+/-2,111$ & $-90,22$ \\
\hline \multicolumn{7}{|l|}{ Cobertura solapada } \\
\hline \multicolumn{7}{|l|}{ Cobertura P } \\
\hline Multiestratificado & $1,603+/-0,576$ & $2,263+/-0,765$ & $3,174+/-0,721$ & $3,456+/-0,915$ & $1,853+/-0,576$ & 115,60 \\
\hline Arbóreo & $0,680+/-0,294$ & $0,838+/-0,391$ & $1,178+/-0,408$ & $1,351+/-0,484$ & $0,671+/-0,274$ & 98,68 \\
\hline Subarbóreo & $0,452+/-0,250$ & $0,814+/-0,289$ & $1,190+/-0,380$ & $1,313+/-0,402$ & $0,861+/-0,407$ & 190,49 \\
\hline Arbustivo & $0,139+/-0,119$ & $0,429+/-0,162$ & $0,648+/-0,238$ & $0,820+/-0,322$ & $0,681+/-0,364$ & 489,93 \\
\hline Herbáceo & $0,524+/-0,275$ & $1,005+/-0,417$ & $1,522+/-0,380$ & $1,575+/-0,436$ & $1,051+/-0,432$ & 200,57 \\
\hline \multicolumn{7}{|l|}{ Cobertura N } \\
\hline Multiestratificado & $0,274+/-0,143$ & $0,318+/-0,156$ & $0,289+/-0,123$ & $0,252+/-0,133$ & $-0,022+/-0,088$ & $-8,03$ \\
\hline Subarbóreo & $0,054+/-0,065$ & $0,096+/-0,083$ & $0,110+/-0,083$ & $0,105+/-0,073$ & $0,052+/-0,055$ & 96,30 \\
\hline Arbustivo & $0,006+/-0,016$ & $0,002+/-0,001$ & $0,021+/-0,035$ & $0,033+/-0,071$ & $0,027+/-0,075$ & 450,00 \\
\hline Herbáceo & $0,217+/-0,126$ & $0,228+/-0,131$ & $0,184+/-0,081$ & $0,147+/-0,086$ & $-0.070+/-0,098$ & $-322,58$ \\
\hline
\end{tabular}


Tabla 4. Datos equivalentes a los expresados en la tabla $n^{\circ} 3$, pero referidos a los 3 tipos sucesionales de especies (regresivas: $\mathrm{R}$; neutras: N; progresivas: P) tanto dentro de cada estrato como en los inventarios multiestratificados (continuación)

\begin{tabular}{|l|c|c|c|c|c|c|}
\hline \multirow{2}{*}{} & \multicolumn{7}{|c|}{ Año } & \multicolumn{2}{c|}{ Variación 2014-1984 } \\
\cline { 2 - 7 } & 1984 & 1994 & 2004 & 2014 & Neta & $\%$ Incr. \\
\hline Cobertura solapada & & & & & & \\
\hline Cobertura R & & & & & & \\
\hline Multiestratificado & $0,182+/-0,124$ & $0,115+/-0,089$ & $0,043+/-0,039$ & $0,012+/-0,021$ & $-0,170+/-0,115$ & $-93,41$ \\
Arbóreo & $0,033+/-0,070$ & $0,033+/-0,070$ & $0,006+/-0,016$ & $0,005+/-0,016$ & $-0,028+/-0,056$ & $-84,85$ \\
Subarbóreo & $0,016+/-0,024$ & $0,011+/-0,021$ & $0,005+/-0,016$ & $0,0004+/-0,0005$ & $-0,015+/-0,024$ & $-93,75$ \\
Arbustivo & $0,038+/-0,033$ & $0,032+/-0,025$ & $0,011+/-0,021$ & $0,0004+/-0,0005$ & $-0,038+/-0,033$ & $-100,00$ \\
Herbáceo & $0,108+/-0,084$ & $0,039+/-0,024$ & $0,022+/-0,025$ & $0,006+/-0,015$ & $-0,102+/-0,082$ & $-94,44$ \\
\hline
\end{tabular}

No se registraron especies $\mathrm{N}$ en el estrato arbóreo. Excepcionalmente, por el bajo valor registrado, los promedios de cobertura solapada inferiores a 0,001 y sus respectivas desviaciones se han expresado con 4 decimales.

términos que serían previsibles conforme al conocimiento fitosociológico. Las especies del tipo $\mathrm{N}$ tienen un comportamiento intermedio, aunque en lo relativo al número de especies presentan una clara dinámica regresiva.

Estos mismos datos quedan claramente corroborados por los valores del estadístico $\mathrm{t}$ al comparar los datos apareados de los diferentes grupos sucesionales dentro de un mismo estrato y para un mismo año -tabla nํ5-. Al resultar más representativo el contraste entre la evolución de las especies progresivas (P) y regresivas (R), la tabla $\mathrm{n}^{\mathrm{o}} 3$ se ha restringido a expresar sólo esos resultados. Nótese que al poseer todas las comparaciones el mismo número de muestras ( $\mathrm{n}=10$ inventarios) y grados de libertad (g.d.l. = 9) la magnitud del valor t nos aproxima al concepto de distancia entre los valores de los grupos de especies progresivas y regresivas. Para todas las series de datos contrastadas, se han encontrado diferencias significativas entre P y R tanto para la riqueza como para la cobertura, pero mientras en la primera dichas diferencias son crecientes con el paso de los decenios, en la cobertura se ve un estancamiento final e incluso un descenso ligero de los valores del estadístico t.

Los resultados obtenidos corroboran la existencia de una evolución aparente dentro de la vegetación, a la que deben añadirse algunos aspectos derivados de las observaciones en campo, no extraíbles de la información sintética aquí aportada, a saber:

- La progresiva abundancia y acumulación de troncos caídos de ejemplares de Pinus, particularmente para la especie $P$. halepensis, subsecuente a la muerte de ejemplares de mayor edad que murieron por muerte natural o por efecto de temporales. 
Tabla 5. Valores del estadístico $t(n=10, g d l=9)$ para el contraste de la media de datos apareados entre los grupos sucesionales $\mathrm{P}$ y $\mathrm{R}$ dentro de cada estrato y en el inventario multiestratificado

\begin{tabular}{|l|c|r|r|r|}
\hline $\begin{array}{l}\text { Valores absolutos de } t \\
\text { por estratos }\end{array}$ & 1984 & 1994 & 2004 & 2014 \\
\hline Riqueza (No especies) & & & & \\
\hline Multiestratificado & $2,952^{*}$ & $13,940^{* * * * *}$ & $20,810^{* * * * *}$ & $29,640^{* * * * *}$ \\
Arbóreo & $6,530^{* * * * *}$ & $5,460^{* * * *}$ & $6,659^{* * * * *}$ & $9,462^{* * * * *}$ \\
Subarbóreo & $15,540^{* * * * *}$ & $17,090^{* * * * *}$ & $23,910^{* * * * *}$ & $32,960^{* * * * *}$ \\
Arbustivo & $6,874^{* * * * *}$ & $13,300^{* * * * *}$ & $23,720^{* * * * *}$ & $29,190^{* * * * *}$ \\
Herbáceo & $3,617^{* * *}$ & $15,370^{* * * * *}$ & $23,160^{* * * * *}$ & $30,420^{* * * * *}$ \\
Cobertura solapada & & & & \\
Multiestratificado & $6,910^{* * * * *}$ & $8,996^{* * * * *}$ & $13,530^{* * * * *}$ & $12,020^{* * * * *}$ \\
Arbóreo & $6,974^{* * * * *}$ & $6,868^{* * * * *}$ & $9,160^{* * * * *}$ & $8,859^{* * * * *}$ \\
Subarbóreo & $5,563^{* * * *}$ & $8,793^{* * * * *}$ & $9,913^{* * * * *}$ & $10,320^{* * * * *}$ \\
Arbustivo & $2,408^{* *}$ & $7,927^{* * * * *}$ & $8,390^{* * * * *}$ & $8,058^{* * * * *}$ \\
Herbáceo & $4,050^{* * *}$ & $7,418^{* * * *}$ & $12,110^{* * * * *}$ & $11,450^{* * * * *}$ \\
\hline
\end{tabular}

Notación: Medias significativamente diferentes para $\left.p=0,05(*), 0.01\left(^{* *}\right), 0,001{ }^{* * *}\right) \circ 0,0001(* * * *)$.

Cabe recordar que la zona se repobló a principios del siglo XX con estas especies, por lo que los ejemplares más ancianos que quedan alcanzan ya edades para las que suele ser esperable la muerte natural en masas cerradas en la zona valenciana.

- La reducción creciente de presencia y cobertura de las especies regresivas más relevantes en el estrato arbóreo, en particular las de Pinus halepensis y P. pinaster subsp. escarena, frente a las progresivas como Q. faginea y Q. ilex subsp. rotundifolia. La tabla no 6 muestra los valores presentados por estas 4 especies en el estrato arbóreo, y la fig. no 8 permite visualizar mediante gráficas estos mismos datos.

\section{Discusión}

Los datos recogidos, sintetizados a través de dos indicadores -riqueza específica y cobertura solapada- evidencian una progresiva evolución de la vegetación donde, con matices puntuales en algunos casos de estratos o de paso entre decenios, tiende a generarse un incremento de ambas variables. La riqueza en especies tiende a presentar ya un ligero descenso en los muestreos más recientes -fig. ํㅜㄱ 7-, lo que podría indicar ya una estabilización propia del paso desde la fresneda hasta la etapa final espe- 
Tabla 6. Datos de la evolución de las especies más representativas del grupo R (Pinus halepensis y $P$. pinaster subsp. escarena) y del grupo $\mathrm{P}$ (Quercus faginea y Q. ilex subsp. rotundifolia) en el estrato arbóreo de las 10 parcelas estudiadas

\begin{tabular}{|c|c|c|c|c|}
\hline & 1984 & 1994 & 2004 & 2014 \\
\hline \multicolumn{5}{|l|}{$\begin{array}{l}\text { № inventarios } \\
\text { con presencia }\end{array}$} \\
\hline Pinus halepensis & 7 & 7 & 4 & 2 \\
\hline $\begin{array}{l}\text { Pinus pinaster } \\
\text { subsp. escarena }\end{array}$ & 4 & 4 & 3 & 2 \\
\hline Quercus faginea & 1 & 2 & 5 & 6 \\
\hline $\begin{array}{l}\text { Quercus ilex } \\
\text { subsp. rotundifolia }\end{array}$ & 2 & 3 & 7 & 10 \\
\hline \multicolumn{5}{|c|}{ Cobertura media (en \%) } \\
\hline Pinus halepensis & $2,790+/-5,562$ & $2,790+/-5,562$ & $0,530+/-1,571$ & $0,510+/-1,578$ \\
\hline $\begin{array}{l}\text { Pinus pinaster } \\
\text { subsp. escarena }\end{array}$ & $0,530+/-1,571$ & $0,540+/-1,571$ & $0,030+/-0,048$ & $0,020+/-0,042$ \\
\hline Quercus faginea & $0,500+/-1,581$ & $0,510+/-1,578$ & $2,280+/-5,571$ & $3,270+/-5,521$ \\
\hline $\begin{array}{l}\text { Quercus ilex } \\
\text { subsp. rotundifolia }\end{array}$ & $0,020+/-0,042$ & $1,010+/-2,103$ & $2,030+/-2,556$ & $6,520+/-6,123$ \\
\hline
\end{tabular}

A fin de evitar el exceso de decimales en la tabla, la cobertura media se ha expresado como porcentaje -multiplicando por 100 el valor de cobertura de Tüxen-Ellenberg equivalente al grado de abundancia-dominancia fitosociológica.

rable de encinar con fresno de flor. El mayor repunte de esta variable en muestreos intermedios es fácilmente justificable porque en esos momentos se alcanza la situación en la que, sin producirse aún un colapso severo de las especies regresivas de la comunidad vegetal, ya empieza a haber suficiente representación de las progresivas, y hay una carga significativa de especies neutras -ver tab. no 4-; en los años siguientes los grupos N y R sufren un descenso progresivo, especialmente a partir de 2004. Para la cobertura solapada los valores son similares aunque los ritmos de descenso de valores en $\mathrm{N}$ y $\mathrm{R}$ no resulten aparentemente tan acusados, e incluso para algunos de los estratos se dé un ligero incremento de coberturas -resultado del crecimiento vegetativo de las especies neutras de implantación tardía, pero sin que se de en paralelo un aumento del número de especies de ese grupo.

Los valores obtenidos para la comparación de dinámicas interdecenales entre las especies más significativas de Pinus (tipo R) y Quercus (tipo P) en el estrato arbóreo - tab. n⿳o 6 y fig, no 8-, demuestran además un avance en el proceso sucesional, aunque para el tiempo transcurrido entre los muestreos inicial y final -30 años-, no puede hablarse 

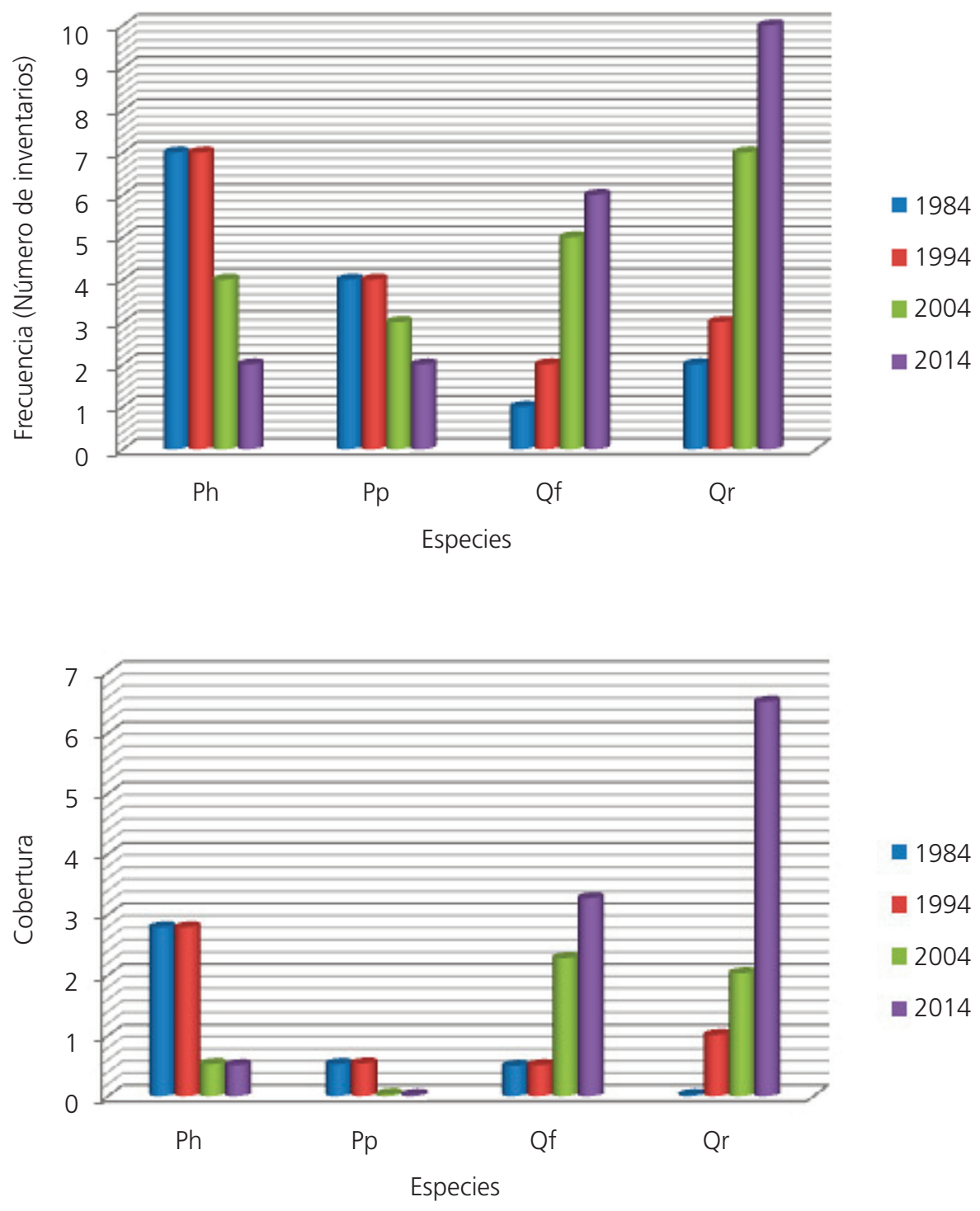

Figura 8. Valores de la frecuencia (número de inventarios con presencia, sobre $n=10$ ) y cobertura media (expresada en \%) en el estrato arbóreo para los 4 años de toma de datos, de las especies más representativas de los grupos sucesionales regresivo

(Ph: Pinus halepensis, Pp: Pinus pinaster subsp. escarena) y regresivo (Qf: Quercus faginea, Qr: Q. ilex subsp. rotundifolia). 
de una sucesión vegetal rápida, lo que en parte podría justificarse por la escasa producción y dispersión local de frutos de las especies de Quercus (E. Laguna, obs. pers.). Los datos tampoco permiten considerar que se entra ya en la fase del encinar propiamente dicho, ya que la cobertura media de Quercus ilex subsp. rotundifolia para las 10 parcelas en el último año de muestreo sólo alcanza el 6,5\%, cuando lo óptimo sería alcanzar el 50\% -es decir, que la encina fuera claramente dominante- o al menos el equivalente al valor 3 de abundancia-dominancia (37,50\%) compensando la diferencia hasta ese 50\% la cobertura de Q. faginea. No obstante, el colapso de las especies regresivas resulta evidente y como simple ejemplo bastaría comparar las sumas de valores de las 2 especies de Pinus y su equivalente de Quercus para los 2 parámetros indicados; de una proporción Pinus vs. Quercus con valor 11 vs. 3 para la frecuencia en 1984 se pasa a 4 vs. 16 en 2014, y si lo analizado son las coberturas, se pasa de 3,32 vs. 0,52 a 0,53 vs. 9,79.

Los resultados obtenidos pueden permitir análisis más detallados o aplicados a otros métodos, especialmente aquellos que pueden aplicarse para prever la evolución de la vegetación. En este aspecto, y considerando algunas innovaciones aquí abarcadas como la diferenciación entre grupos de especies en función de su valor como indicadores de etapas concretas de dicha evolución, pueden aplicarse al testado de la efectividad del método "interactividad Fitoindicación/Fitoacción" propuesto por Meaza y Cuesta (2010) y Gómez Montblanch y Meaza (2010), abreviado con el acrónimo Fi/Fa, que se ha aplicado hasta ahora a diversos tipos de comunidades arbóreas, directamente (Meaza et al., 2011; Gómez Montblanch y Meaza, 2011), o combinado con otras metodologías como ocurre con el método de Gentry para la estimación de la estructura de comunidades vegetales leñosas (Gómez Montblanch et al., 2010; Gómez Montblanch y Meaza, 2013) o el de los valores indicadores de Ellenberg (Gómez Montblanch et al., 2014).

\section{Agradecimientos}

Al Instituto de Estudios Comarcales Hoya de Buñol-Chiva por permitir la reproducción de los dibujos y mapas, extraídos parcialmente de la obra de Laguna (1997); id. al Servicio de Vida Silvestre de la Generalitat Valenciana por el permiso de reproducción de dibujo de Laguna (1990). A los Dres. Miguel Guara y Rafael Currás (Depto. de Botánica, Facultad de Ciencias Biológicas de la Universitat de València) por su asesoramiento en el desarrollo de los primeros muestreos de las parcelas de la zona de estudio.

\section{Bibliografía}

Ballester, G. y Stübing, G., 1990. Sierra del Carrascal de Alcoi: Flora y vegetación. Alicante, Caja de Ahorros Provincial de Alicante.

Braun-Blanquet, J., 1979. Fitosociología. Bases para el estudio de las comunidades vegetales. Madrid, Blume. 
Cardona, M.A., 1980. Funcionalisme i ecologia d'algunes comunitats vegetals barcelonines. Barcelona, Institut d'Estudis Catalans.

Castroviejo, S. (coord. edit.), 1986-2014. Flora iberica. Plantas vasculares de la Península Ibérica e Islas Baleares. 19 vols, Madrid, CSIC-Real Jardín Botánico.

Costa, M., 1986. La vegetación del País Valenciano. Valencia, Universitat de València.

Costa, M., 1999. La vegetación y el paisaje en las tierras valencianas. Madrid, Rueda.

Costa, M., Peris, J.B. y Stübing, G., 1987. Hedero helicis-Quercetum rotundifoliae: Una nueva serie de vegetación valenciano-tarraconense. Lazaroa, 7, 85-91.

Gómez Montblanch, D.C. y Meaza, G., 2010. Nueva perspectiva metodológica en la investigación biogeográfica-geodinámica: La propuesta evaluativa FI/FA. En Giménez, P., Marco, J.A., Matarredona, E., Padilla, A. y Sánchez Pardo, A. (eds.). Biogeografía, una ciencia para la conservación del medio, Alicante, Universidad de Alicante, p. 81-90.

Gómez Montblanch, D.C. y Meaza, G., 2011. Interactividad fitoindicación /fitoacción. Aplicación en fresnedas de los ríos Cerneja (Burgos) y Guadaíra (Sevilla). Lurralde: Investigación y Espacio, , 33, 15-36.

Gómez Montblanch, D.C. y Meaza, G., 2013. Funcionalidad geoecológica del tarajal del Arroyo del Aceitero (Alto Guadaíra, Sevilla). Lurralde: Investigación y Espacio, 36: 51-66.

Gómez Montblanch, D., Meaza, G. y Cámara, R., 2010. Aplicación del método FI/FA al bosque de ribera del arroyo de la Madre (Cuenca del Guadaíra, Sevilla). En Giménez, P., Marco, J.A., Matarredona, E., Padilla, A. y Sánchez Pardo, A. (eds.). Biogeografía, una ciencia para la conservación del medio, Alicante, Universidad de Alicante, p. 233-244.

Gómez Montblanch, D., Laguna, E. y Meaza, G., 2014. Caracterización geoecológica de comunidades vegetales a partir de valores indicadores de Ellenberg y del método Fitoindicación/Fitoacción. Ensayo de aplicación en los tarajales del Arroyo Aceitero (Cuenca del Guadaíra, Sevilla). Geographicalia, 65, 87-114.

Hammer, Ø., 2014. PAST 3.x - the Past of the future. Paquete estadistico, Oslo, Univerity of Oslo, Natural History Museum, http://folk.uio.no/ohammer/past/index.html (Consulta: 10.11.2014).

Hammer, Ø., Harper, D.A.T. y Ryan, P.D., 2001. PAST: Palaeontological statistics software package for education and data analysis. Palaeontologia electronica 4(1): 1-9. http://palaeo-electronica.org/2001_1/past/issue1_01.htm (Consulta: 10.11.2014).

IUSS Grupo de Trabajo WRB., 2007. Base Referencial Mundial del Recurso Suelo. Primera actualización 2007. Informes sobre Recursos Mundiales de Suelos ํํ103. Roma, FAO.

Laguna, E., 1990. Bases para la gestión de los carrascales valencianos, III. Tiempos y espacios para la revegetación y aspectos ecofisiológios básicos. Medi Natural, 2, 67-78.

Laguna, E., 1995. Fenología de la flora y vegetación de la serie del carrascal basófilo mesomediterráneo en la umbría del Fresnal de Buñol (Sierra de Malacara, Valencia). Valencia, Universitat de València, Servei de Publicacions, Serie Tesis Doctorales en Microfichas, nº 055-21.

Laguna, E., 1997. Vegetación y flora de la Umbia del Fresnal (Sierra de Malacara, Hoya de Buñol-Chiva). Buñol (Valencia), Instituto de Estudios Comarcales Hoya de Buñol-Chiva. 
Laguna, E., 2012. El método fitosociológico aplicado al seguimiento de la evolución de la flora en espacios protegidos: el caso de Limonium dufourii en la microrreserva de flora 'Cap de Cullera' (Valencia). En Cunill, R., Pélachs, A., Pérez-Obiol, R. y Soriano, J.M. (eds.). Las zonas de montaña: Gestión y Biodiversidad. VII Congreso Español de Biogeografía. Barcelona, Universitat Autònoma de Barcelona, Departament de Geografia, p. 262-268.

Laguna, E. y Pérez Rocher, B., 1998. La vegetación potencial de la Sierra de Malacara (Hoya de Buñol-Chiva). Revista de Estudios Comarcales Hoya de Buñol-Chiva, 3, 243-250.

Laguna, E. y Ferrer-Gallego, P.P., 2015. Propuestas de aplicación de los índices de diversidad para usos taxonómicos, fitosociológicos y listas rojas de flora amenazada. Flora Montiberica, 60, 18-31.

Mateo, G. y Crespo, M.B., 2014. Claves ilustradas para la flora valenciana. Jaca, Jolube Consultor y Editor Botánico. Jaca.

Mateo, G., Crespo M.B. y Laguna, E. (eds.), 2011-2015. Flora Valentina. 3 vols. Valencia, Fundación de la Comunidad Valenciana para el Medio Ambiente.

Meaza, G. y Cuesta, M.J., 2010. Fitoindicación/fitoacción ambiental y territorial. Ensayo de aplicación en la Reserva de la Biosfera de Urdaibai (País Vasco). Boletín de la AGE, 54, 99-123.

Meaza, G., Alonso, M., García Codrón, J.C., García Abad, J.J., Gómez Montblanch, D.C., Gurrutxaga, M.; López, D. y Represa, A., 2011. Aplicación de la metodología fitoindicación/fitoacción a diversas comunidades vegetales del entorno de Espinosa de los Monteros (Burgos). En Cadiñanos, J.A., Lozano, P.J., Gurrutxaga, M., Varela, R., Fernández Perianes, P., Lozano, M.A. y Martínez Montecello, A. (coord.). Paisajes de transición y gradientes biogeográficos. Bilbao, Universidad del País Vasco, Servicio Editorial, p. 97-115.

Ozenda, P., 1964. Biologie Végétale: Biogéographie Végétale. Paris, Doin.

Pintó, J., Meaza, G. y Beltrán, E., 2000. Dinámica de la vegetación. En Meaza, G. (coord.). Metodología y práctica de la Biogeografía. Barcelona, Ediciones del Serbal, p. 147-197.

Rivas-Martínez, S., 2007. Mapa de series, geoseries y geopermaseries de vegetación de España [Memoria del Mapa de Vegetación Potencial de España, parte 1]. Itinera Geobotanica n.s., 17, 1-436.

Rivas-Martínez, S., Díaz, T.E., Fernández-González, F., Izco, J., Loidi, J., Lousa, M. y Penas, A., 2001. Vascular plant communities of Spain and Portugal. Addenda to the syntaxonomical checklist of 2001, Part I. Itinera Geobotanica, 15(1), 5-432.

Sokal, R.R. y Rohlf, F.J., 1994. Biometry: The principles and practices of statistics in biological research (3르. ed.). Nueva York, W.H. Freeman. 


\section{Anexo 1. Relación de especies y asignación a grupos sucesionales}

Regresivas (R): Asphodelus ramosus, Biscutella stenophylla subsp. stenophylla, Brachypodium retusum, Centaurea setabensis, Cuscuta epithymum, Daphne gnidium, Dorycnium pentaphyllum pentaphyllum, Echinops ritro, Erica multiflora, Eryngium campestre, Euphorbia characias, E. flavicoma, E. nicaeensis, Genista scorpius, Helianthemum croceum cavanillesianum, H. rotundifolium, Helichrysum italicum subsp. serotinum, Helictotrichon filifolium, Juniperus phoenicea, Lavandula latifolia, Lithodora fruticosa, Phlomis crinita, Pinus halepensis, Pinus pinaster escarena, Rosmarinus officinalis, Rostraria cristata, Sanguisorba verrucosa, Satureja intricata subsp. gracilis, Sedum sediforme subsp. sediforme, Sideritis incana, Staehelina dubia, Thymus piperella, Th. vulgaris subsp. vulgaris, Ulex parviflorus.

Neutras (N): Anethum graveolens, Aphyllanthes monspeliensis, Aristolochia pistolochia, Aster sedifolius, Avenula bromoides, Bupleurum fruticescens, Bupleurum rigidum, Carex halleriana, Centaurium quadrifolium, Clinopodium alpinum subsp. meridionalis, Coronilla minima subsp. lotoides, Crepis albida subsp. scorzoneroides, Dactylis glomerata subsp. hispanica, Dictamnus bispanicus, Erinacea anthyllis, Erophila verna, Erysimum gomezcampoi, Ficus carica, Galium maritimum, G. parisiense, G. setaceum, Globularia linifolia subsp. linifolia, Guillonea scabra, Hornungia petraea, Hypericum perforatum, Juniperus oxycedrus, Knautia subscaposa, Lathyrus pulcher, Linum narbonense, Melica minuta subsp. minuta, Ophrys arnoldi, Phillyrea angustifolia, Pistacia terebinthus, Quercus coccifera, Ranunculus gramineus, Rhamnus oleoides subsp. angustifolia, Rubia peregrina subsp. peregrina, Scabiosa turolensis, Teucrium chamaedrys subsp. pinnatifidum, Thalictrum tuberosum, Vicia sativa subsp. nigra.

Progresivas (P): Acer opalus subsp. granatense, Adonis vernalis, Amelanchier ovalis, Arabis planisiliqua, Arbutus unedo, Aristolochia paucinervis, Arbenatherum elatius subsp. sardoum, Asparagus acutifolius, Brachypodium phoenicoides, Bunium balearicum, Cardamine hirsuta, Carex flacca subsp. serrulata, C. humilis, Catananche coerulea, Cephalanthera damasonium, Conopodium arvense, Crataegus monogyna, Teline patens (=Cytisus patens), Festuca arundinacea subsp. fenas, F. capillifolia, Filipendula vulgaris, Fraxinus ornus, Geranium prupureum, Hedera helix subsp. rhizomatifera, Inula conyza, I. helenioides, Leucanthemum gracilicaule, Lonicera implexa f. velutina, L. implexa var. valentina, Ononis aragonensis, Orobanche hederae, Osyris alba, Pimpinella espanensis, P. tragium subsp. litophila, Piptatherum paradoxum, Poa compressa, P. pratensis, Prunella laciniata, Quercus faginea, Quercus ilex subsp. rotundifolia, Quercus $\times$ auzandrii nothosubsp. agrifolia, $Q . \times$ battandieri, Rhamnus alaternus, Rosa agrestis, R. micrantha, R. pouzinii, Rubus ulmifolius, Ruscus aculeatus, Saponaria ocymoides, Scrophularia tanacetifolia, Smilax aspera, Stachys heraclea, Tanacetum corymbosum, Thlaspi perfoliatum, Th. stenopterum, Viburnum tinus, Vicia onobrychioides, V. villosa, Viola alba, V. kitaibeliana, V. riviniana, V. rupestris. 
Anexo 2. Valores de frecuencia y cobertura media obtenidos para cada una de las 137 especies presentes en los inventarios. La frecuencia expresa el número de inventarios en que la especie está presente. La cobertura se expresa como el promedio del valor de cobertura media (en porcentaje) de Tüxen-Ellenberg de los 10 inventarios. Sobreimpresionados en las frecuencias, en fondo gris oscuro se resaltan los casos con presencia en al menos el 50\% de los inventarios; en gris claro, presencia en al menos el 30\%. En las coberturas, en gris oscuro, casos con coberturas medias iguales o superiores al 10\%; en gris claro, id. al 5\%.

\begin{tabular}{|c|c|c|c|c|c|c|c|c|}
\hline \multirow[b]{2}{*}{ Especies } & \multicolumn{4}{|c|}{ Frecuencia ( $n^{\circ}$ inventarios) } & \multicolumn{4}{|c|}{ Cobertura media (\%) } \\
\hline & 1984 & 1994 & 2004 & 2014 & 1984 & 1994 & 2004 & 2014 \\
\hline Acer opalus granatensis & 5 & 7 & 6 & 7 & 11,50 & 13,50 & 16,76 & 24,76 \\
\hline Adonis vernalis & 1 & 1 & 1 & 0 & 0,01 & 0,01 & 0,01 & 0,00 \\
\hline Amelanchier ovalis & 9 & 10 & 10 & 10 & 5,26 & 10,26 & 13,25 & 12,01 \\
\hline Anethum graveolens & 1 & 0 & 0 & 0 & 0,01 & 0,00 & 0,00 & 0,00 \\
\hline Aphyllanthes monspeliensis & 4 & 4 & 4 & 2 & 0,04 & 0,04 & 0,04 & 0,02 \\
\hline Arabis planisiliqua & 0 & 0 & 1 & 2 & 0,00 & 0,00 & 0,01 & 0,02 \\
\hline Arbutus unedo & 3 & 4 & 4 & 4 & 0,03 & 0,52 & 1,01 & 0,53 \\
\hline Aristolochia paucinervis & 1 & 2 & 2 & 2 & 0,01 & 0,02 & 0,02 & 0,02 \\
\hline Aristolochia pistolochia & 1 & 1 & 2 & 0 & 0,01 & 0,01 & 0,02 & 0,00 \\
\hline Arrhenatherum elatius sardoum & 9 & 10 & 10 & 10 & 2,54 & 3,53 & 7,50 & 7,01 \\
\hline Asparagus actifolius & 5 & 7 & 9 & 9 & 1,52 & 1,54 & 1,06 & 2,54 \\
\hline Asphodelus ramosus & 7 & 6 & 1 & 0 & 1,05 & 0,55 & 0,01 & 0,00 \\
\hline Aster sedifolius & 0 & 1 & 1 & 0 & 0,00 & 0,01 & 0,01 & 0,00 \\
\hline Avenula bromoides & 3 & 4 & 3 & 2 & 0,03 & 0,04 & 0,03 & 0,02 \\
\hline Biscutella stenophylla stenophylla & 4 & 0 & 0 & 0 & 0,04 & 0,00 & 0,00 & 0,00 \\
\hline Brachypodium phoenicoides & 10 & 10 & 10 & 10 & 17,75 & 21,75 & 22,25 & 21,00 \\
\hline Brachypodium retusum & 10 & 10 & 10 & 9 & 7,01 & 3,04 & 2,06 & 0,58 \\
\hline Bunium balearicum & 0 & 0 & 1 & 2 & 0,00 & 0,00 & 0,01 & 0,02 \\
\hline Bupleurum fruticescens & 5 & 3 & 1 & 0 & 0,54 & 0,03 & 0,01 & 0,00 \\
\hline Bupleurum rigidum & 7 & 6 & 6 & 4 & 3,28 & 0,55 & 0,55 & 0,04 \\
\hline Cardamine birsuta & 0 & 1 & 1 & 1 & 0,00 & 0,01 & 0,01 & 0,01 \\
\hline Carex flacca serrulata & 6 & 7 & 7 & 7 & 4,25 & 3,77 & 2,03 & 1,05 \\
\hline Carex halleriana & 9 & 9 & 9 & 10 & 4,77 & 5,26 & 5,75 & 6,51 \\
\hline
\end{tabular}




\begin{tabular}{|c|c|c|c|c|c|c|c|c|}
\hline \multirow[b]{2}{*}{ Especies } & \multicolumn{4}{|c|}{ Frecuencia ( $n^{o}$ inventarios) } & \multicolumn{4}{|c|}{ Cobertura media (\%) } \\
\hline & 1984 & 1994 & 2004 & 2014 & 1984 & 1994 & 2004 & 2014 \\
\hline Carex humilis & 6 & 8 & 10 & 10 & 3,76 & 5,52 & 6,03 & 6,79 \\
\hline Catananche caerulea & 1 & 7 & 5 & 3 & 0,01 & 0,07 & 0,55 & 0,52 \\
\hline Centaurea setabensis & 1 & 0 & 0 & 0 & 0,01 & 0,00 & 0,00 & 0,00 \\
\hline Centaurium quadrifolium & 1 & 0 & 0 & 0 & 0,01 & 0,00 & 0,00 & 0,00 \\
\hline Cephalanthera damasonium & 0 & 0 & 0 & 1 & 0,00 & 0,00 & 0,00 & 0,01 \\
\hline Clinopodium alpinum meridionalis & 0 & 1 & 0 & 0 & 0,00 & 0,01 & 0,00 & 0,00 \\
\hline Conopodium arvense & 0 & 1 & 0 & 4 & 0,00 & 0,01 & 0,00 & 0,53 \\
\hline Coronilla minima lotoides & 2 & 1 & 0 & 0 & 0,02 & 0,01 & 0,00 & 0,00 \\
\hline Crataegus monogyna & 2 & 7 & 8 & 9 & 0,51 & 1,01 & 2,01 & 2,54 \\
\hline Crepis albida scorzoneroides & 1 & 2 & 3 & 3 & 0,50 & 0,02 & 0,03 & 0,03 \\
\hline Cuscuta epithymum & 0 & 1 & 0 & 0 & 0,00 & 0,01 & 0,00 & 0,00 \\
\hline Cytisus heterochrous (=Teline patens) & 10 & 10 & 10 & 10 & 20,25 & 32,00 & 31,50 & 21,50 \\
\hline Dactylis glomerata bispanica & 1 & 2 & 2 & 0 & 0,01 & 0,02 & 0,02 & 0,00 \\
\hline Daphne gnidium & 8 & 8 & 2 & 0 & 0,08 & 0,08 & 0,02 & 0,00 \\
\hline Dictamnus hispanicus & 1 & 1 & 1 & 1 & 0,01 & 0,01 & 0,01 & 0,01 \\
\hline Dorycnium pentaphyllum pentaphyllum & 2 & 2 & 0 & 0 & 0,02 & 0,02 & 0,00 & 0,00 \\
\hline Echinops ritro & 3 & 2 & 0 & 0 & 0,03 & 0,02 & 0,00 & 0,00 \\
\hline Erica multiflora & 5 & 3 & 0 & 0 & 1,03 & 1,03 & 0,00 & 0,00 \\
\hline Erinacea anthyllis & 2 & 3 & 3 & 2 & 1,00 & 0,52 & 0,03 & 0,02 \\
\hline Erophila verna & 0 & 2 & 2 & 1 & 0,00 & 0,02 & 0,02 & 0,01 \\
\hline Eryngium campestre & 2 & 0 & 0 & 0 & 0,02 & 0,00 & 0,00 & 0,00 \\
\hline Erysimum gomezcampoi & 1 & 2 & 0 & 0 & 0,01 & 0,02 & 0,00 & 0,00 \\
\hline Euphorbia characias & 0 & 1 & 1 & 1 & 0,00 & 0,01 & 0,01 & 0,01 \\
\hline Euphorbia flavicoma & 5 & 2 & 0 & 0 & 0,05 & 0,02 & 0,00 & 0,00 \\
\hline Euphorbia nicaeensis & 2 & 2 & 0 & 0 & 0,02 & 0,02 & 0,00 & 0,00 \\
\hline Festuca arundinacea fenas & 3 & 8 & 8 & 9 & 1,50 & 1,55 & 3,02 & 1,56 \\
\hline Festuca capillifolia & 3 & 7 & 9 & 9 & 2,75 & 3,28 & 5,53 & 6,29 \\
\hline Ficus carica v. capraficus & 1 & 1 & 1 & 1 & 0,01 & 0,01 & 0,01 & 0,01 \\
\hline Filipendula vulgaris & 0 & 1 & 1 & 1 & 0,00 & 0,01 & 0,01 & 0,01 \\
\hline Fraxinus ornus & 10 & 10 & 10 & 10 & 32,75 & 43,50 & 67,50 & 67,50 \\
\hline
\end{tabular}




\begin{tabular}{|c|c|c|c|c|c|c|c|c|}
\hline \multirow[b]{2}{*}{ Especies } & \multicolumn{4}{|c|}{ Frecuencia (no inventarios) } & \multicolumn{4}{|c|}{ Cobertura media (\%) } \\
\hline & 1984 & 1994 & 2004 & 2014 & 1984 & 1994 & 2004 & 2014 \\
\hline Galium maritimum & 2 & 2 & 3 & 5 & 0,02 & 1,76 & 0,52 & 0,54 \\
\hline Galium parisiense & 4 & 5 & 4 & 2 & 0,04 & 0,05 & 0,53 & 0,02 \\
\hline Galium setaceum & 0 & 0 & 1 & 0 & 0,00 & 0,00 & 0,01 & 0,00 \\
\hline Galium valentinum & 3 & 1 & 0 & 0 & 0,03 & 0,01 & 0,00 & 0,00 \\
\hline Genista scorpius & 1 & 1 & 0 & 0 & 0,01 & 0,01 & 0,00 & 0,00 \\
\hline Geranium purpureum & 3 & 7 & 9 & 9 & 2,26 & 4,04 & 4,55 & 3,79 \\
\hline Globularia linifolia linifolia & 3 & 2 & 0 & 0 & 0,03 & 0,02 & 0,00 & 0,00 \\
\hline Guillonea scabra & 2 & 5 & 4 & 1 & 0,51 & 0,05 & 0,04 & 0,01 \\
\hline Hedera helix rbizomatifera & 8 & 10 & 10 & 10 & 23,25 & 25,26 & 29,50 & 38,50 \\
\hline Helianthemum croceum cavanillesianum & 1 & 0 & 0 & 0 & 0,01 & 0,00 & 0,00 & 0,00 \\
\hline Helianthemum rotundifolium & 3 & 0 & 0 & 0 & 0,52 & 0,00 & 0,00 & 0,00 \\
\hline Helichrysum italicum serotinum & 1 & 0 & 0 & 0 & 0,01 & 0,00 & 0,00 & 0,00 \\
\hline Helictotrichon filifolium & 5 & 4 & 3 & 0 & 0,54 & 0,04 & 0,03 & 0,00 \\
\hline Hornungia petraea & 0 & 1 & 1 & 1 & 0,00 & 0,01 & 0,01 & 0,01 \\
\hline Hypericum perforatum & 1 & 2 & 0 & 0 & 0,01 & 0,02 & 0,00 & 0,00 \\
\hline Inula conyza & 1 & 3 & 3 & 2 & 0,01 & 0,03 & 0,03 & 0,02 \\
\hline Inula belenioides & 3 & 3 & 3 & 6 & 0,52 & 0,52 & 0,52 & 0,06 \\
\hline Juniperus oxycedrus & 6 & 5 & 4 & 2 & 0,54 & 0,54 & 0,53 & 0,02 \\
\hline Juniperus phoenicea & 7 & 6 & 4 & 3 & 1,54 & 1,04 & 0,53 & 0,03 \\
\hline Knautia subscaposa & 0 & 0 & 2 & 0 & 0,00 & 0,00 & 0,02 & 0,00 \\
\hline Lathyrus pulcher & 2 & 6 & 5 & 4 & 1,00 & 1,04 & 0,54 & 0,04 \\
\hline Lavandula latifolia & 7 & 4 & 0 & 0 & 0,07 & 0,04 & 0,00 & 0,00 \\
\hline Leucanthemum gracilicaule & 0 & 2 & 4 & 3 & 0,00 & 0,02 & 0,53 & 0,53 \\
\hline Linum narbonense & 1 & 1 & 0 & 0 & 0,01 & 0,01 & 0,00 & 0,00 \\
\hline Litbodora fruticosa & 2 & 0 & 0 & 0 & 0,02 & 0,00 & 0,00 & 0,00 \\
\hline Lonicera implexa f. velutina & 5 & 5 & 7 & 6 & 1,79 & 2,77 & 2,77 & 2,02 \\
\hline Lonicera implexa v. valentina & 7 & 9 & 10 & 10 & 1,05 & 3,03 & 5,76 & 7,50 \\
\hline Melica minuta minuta & 3 & 5 & 3 & 3 & 1,01 & 0,05 & 0,03 & 0,03 \\
\hline Ononis aragonensis & 4 & 6 & 7 & 7 & 4,01 & 5,01 & 3,76 & 2,52 \\
\hline Ophrys arnoldii & 1 & 1 & 1 & 0 & 0,01 & 0,01 & 0,01 & 0,00 \\
\hline
\end{tabular}




\begin{tabular}{|c|c|c|c|c|c|c|c|c|}
\hline \multirow[b]{2}{*}{ Especies } & \multicolumn{4}{|c|}{ Frecuencia ( $n^{o}$ inventarios) } & \multicolumn{4}{|c|}{ Cobertura media (\%) } \\
\hline & 1984 & 1994 & 2004 & 2014 & 1984 & 1994 & 2004 & 2014 \\
\hline Orobanche hederae & 0 & 0 & 4 & 3 & 0,00 & 0,00 & 0,04 & 1,50 \\
\hline Osyris alba & 1 & 3 & 3 & 4 & 0,01 & 0,03 & 0,03 & 0,04 \\
\hline Phlomis crinita & 1 & 1 & 0 & 0 & 0,01 & 0,01 & 0,00 & 0,00 \\
\hline Pimpinella espanensis & 0 & 1 & 2 & 1 & 0,00 & 0,01 & 0,02 & 0,01 \\
\hline Pimpinella tragium litophila & 0 & 0 & 0 & 1 & 0,00 & 0,00 & 0,00 & 0,01 \\
\hline Pinus halepensis & 7 & 7 & 4 & 2 & 2,79 & 2,79 & 0,53 & 0,51 \\
\hline Pinus pinaster escarena & 4 & 4 & 3 & 2 & 0,53 & 0,53 & 0,03 & 0,02 \\
\hline Piptatherum paradoxum & 1 & 5 & 9 & 10 & 0,50 & 0,54 & 2,54 & 2,55 \\
\hline Pistacia terebinthus & 1 & 1 & 1 & 2 & 0,01 & 0,01 & 0,50 & 0,51 \\
\hline Poa compressa & 3 & 5 & 9 & 10 & 2,75 & 4,02 & 5,04 & 3,80 \\
\hline Poa pratensis & 0 & 1 & 1 & 3 & 0,00 & 0,50 & 0,50 & 0,03 \\
\hline Prunella laciniata & 0 & 0 & 1 & 1 & 0,00 & 0,00 & 0,01 & 0,01 \\
\hline Quercus coccifera & 10 & 10 & 10 & 10 & 4,78 & 9,02 & 10,00 & 10,00 \\
\hline Quercus faginea & 3 & 7 & 8 & 10 & 0,50 & 1,05 & 2,28 & 4,28 \\
\hline Quercus ilex rotundifolia & 3 & 9 & 10 & 10 & 0,03 & 1,01 & 3,04 & 6,52 \\
\hline Quercus $\times$ auzandrii agrifolia & 1 & 1 & 2 & 1 & 0,01 & 0,01 & 0,51 & 1,00 \\
\hline Quercus $\times$ battandieri & 0 & 0 & 0 & 1 & 0,00 & 0,00 & 0,00 & 0,01 \\
\hline Ranunculus gramineus & 2 & 4 & 2 & 2 & 0,02 & 0,54 & 0,02 & 0,02 \\
\hline Rhamnus alaternus & 8 & 8 & 9 & 10 & 2,04 & 6,01 & 6,50 & 6,51 \\
\hline Rhamnus oleoides angustifolia & 2 & 2 & 1 & 0 & 0,02 & 0,02 & 0,01 & 0,00 \\
\hline Rosa agrestis & 1 & 5 & 4 & 5 & 0,01 & 0,51 & 1,01 & 1,03 \\
\hline Rosa micrantha & 2 & 3 & 4 & 5 & 0,02 & 0,51 & 0,53 & 0,54 \\
\hline Rosa pouzinii & 3 & 4 & 5 & 7 & 0,52 & 0,53 & 1,03 & 1,54 \\
\hline Rosmarinus officinalis & 3 & 3 & 1 & 0 & 0,03 & 0,03 & 0,01 & 0,00 \\
\hline Rostraria cristata & 1 & 0 & 0 & 0 & 0,01 & 0,00 & 0,00 & 0,00 \\
\hline Rubia peregrina peregrina & 10 & 10 & 10 & 10 & 2,55 & 6,25 & 7,50 & 6,25 \\
\hline Rubus ulmifolius & 1 & 4 & 7 & 7 & 0,01 & 0,04 & 1,05 & 3,50 \\
\hline Ruscus aculeatus & 2 & 6 & 9 & 10 & 1,00 & 2,77 & 6,27 & 9,02 \\
\hline Sanguisorba verrucosa & 0 & 0 & 1 & 0 & 0,00 & 0,00 & 0,01 & 0,00 \\
\hline Saponaria ocymoides & 4 & 4 & 7 & 4 & 0,01 & 0,04 & 1,05 & 0,04 \\
\hline
\end{tabular}




\begin{tabular}{|c|c|c|c|c|c|c|c|c|}
\hline \multirow[b]{2}{*}{ Especies } & \multicolumn{4}{|c|}{ Frecuencia ( $n^{\circ}$ inventarios) } & \multicolumn{4}{|c|}{ Cobertura media (\%) } \\
\hline & 1984 & 1994 & 2004 & 2014 & 1984 & 1994 & 2004 & 2014 \\
\hline Satureja intricata gracilis & 2 & 3 & 0 & 0 & 0,04 & 0,03 & 0,00 & 0,00 \\
\hline Scabiosa turolensis & 2 & 2 & 0 & 0 & 0,02 & 0,02 & 0,00 & 0,00 \\
\hline Scrophularia tanacetifolia & 0 & 1 & 2 & 0 & 0,00 & 0,01 & 0,02 & 0,00 \\
\hline Sedum sediforme sediforme & 2 & 0 & 0 & 0 & 0,02 & 0,00 & 0,00 & 0,00 \\
\hline Sideritis incana & 1 & 0 & 0 & 0 & 0,01 & 0,00 & 0,00 & 0,00 \\
\hline Smilax aspera & 3 & 3 & 3 & 4 & 0,03 & 0,03 & 0,52 & 1,01 \\
\hline Stachys beraclea & 0 & 1 & 1 & 0 & 0,00 & 0,01 & 0,01 & 0,00 \\
\hline Staebelina dubia & 1 & 0 & 0 & 0 & 0,01 & 0,00 & 0,00 & 0,00 \\
\hline Tanacetum corymbosum & 0 & 1 & 2 & 2 & 0,00 & 0,50 & 0,02 & 0,51 \\
\hline Teucrium chamaerdrys pinnatifidum & 6 & 6 & 1 & 0 & 0,55 & 0,06 & 0,01 & 0,00 \\
\hline Thalictrum tuberosum & 9 & 9 & 10 & 9 & 6,02 & 5,75 & 2,06 & 1,07 \\
\hline Thlaspi perfoliatum & 1 & 2 & 6 & 7 & 0,01 & 0,02 & 0,06 & 0,56 \\
\hline Thlaspi stenopterum & 2 & 4 & 4 & 4 & 0,51 & 0,04 & 0,53 & 0,53 \\
\hline Thymus piperella & 6 & 5 & 1 & 0 & 0,06 & 0,05 & 0,01 & 0,00 \\
\hline Thymus vulgaris vulgaris & 3 & 1 & 0 & 0 & 0,03 & 0,01 & 0,00 & 0,00 \\
\hline Ulex parviflorus & 10 & 10 & 7 & 4 & 2,55 & 2,55 & 1,05 & 0,04 \\
\hline Viburnum tinus & 6 & 10 & 10 & 10 & 8,75 & 10,02 & 20,02 & 32,00 \\
\hline Vicia onobrychioides & 0 & 1 & 1 & 1 & 0,00 & 0,01 & 0,01 & 0,01 \\
\hline Vicia sativa nigra & 0 & 1 & 0 & 0 & 0,00 & 0,01 & 0,00 & 0,00 \\
\hline Vicia villosa & 1 & 1 & 2 & 0 & 0,01 & 0,01 & 0,02 & 0,00 \\
\hline Viola alba & 8 & 10 & 10 & 10 & 3,78 & 7,77 & 17,00 & 16,25 \\
\hline Viola kitaibeliana & 0 & 0 & 1 & 0 & 0,00 & 0,00 & 0,01 & 0,00 \\
\hline Viola riviniana & 1 & 3 & 5 & 6 & 0,01 & 0,52 & 1,03 & 1,04 \\
\hline Viola rupestris & 2 & 5 & 10 & 10 & 0,02 & 0,54 & 2,55 & 3,53 \\
\hline
\end{tabular}


